\title{
A modified social spider algorithm for an efficient data dissemination in VANET
}

\author{
Achyut Shankar ${ }^{1} \cdot$ Rajaguru Dayalan $^{2} \cdot$ Chinmay Chakraborty $^{3}$ (D) \\ Chandramohan Dhasarathan ${ }^{4} \cdot$ Manish Kumar $^{5}$
}

Received: 17 June 2021 / Accepted: 22 November 2021

(c) The Author(s), under exclusive licence to Springer Nature B.V. 2021

\begin{abstract}
Technical growth in the field of communication and information is an important aspect in the development and innovation of industrial automation and in the recent advances in the field of communications. The recent development of mobile communications has led to worldwide ubiquitous information sharing and has rehabilitated human lifestyles. This communication revolution is now introducing effective information sharing into the automotive industry. The current technology is extending this field of applications for vehicle safety, improving the efficiency in traffic management, offering reliable assistance for drivers and supporting the modern field of vehicle design. With these advances, the vehicular network concept has grabbed worldwide attention. In this article, a novel samplingbased estimation scheme (SES), to initiate the involvements and increase the probabilistic contacts of vehicle communication. The scheme is divided into a few segments, for ease of operations with a perfect sample. The contact duration between two vehicles moving in opposite directions on their overlapped road is lower, but their contact probability is higher. By contrast, the duration of the contact between two vehicles moving in the same direction on their overlapped road is higher, but their contact probability is lower. SES can easily obtain efficient routing by considering the above-mentioned stochastic contacts. Furthermore, we investigate the content transmission among the probabilistic contacts, by using the flow model with probabilistic capacities. The performance of the proposed SES is experimentally validated with the probabilistic contacts in VANETs.
\end{abstract}

Keywords Vehicular ad hoc networks · Routing · Quality of service · Clustering · Sampling-based estimation scheme $\cdot$ Modified social spider algorithm

\section{Introduction}

The recent trend in vehicular networks is to provide reliable, viable and security-based information to vehicles to enhance passenger safety and commercial success. For establishing this concept of increased safety and commercial achievements, a standard background

Chinmay Chakraborty

cchakrabarty@bitmesra.ac.in

Extended author information available on the last page of the article 
is required on our roads. A detailed study will be presented in this session for better understanding of vehicular ad hoc network (VANET).

The recent advances in mobile communications have led to the implementation of vehicle-based networks in rural, urban and highway regions based on their requirements. The ultimate aim of such networks is to efficiently deliver messages between vehicles and infrastructure. To achieve this target, VANET uses different networks with respect to the need of the delivering messages. This can be categorized into three forms. Vehicle-to-vehicle ad hoc network (V2V): This network establishes a connection between vehicles for transferring information without depending on any infrastructure. This type of network is mostly used for sharing information regarding accident reports. The two important aspects in this network are efficient message dissemination with respect to time and security.

Vehicle-to-Infrastructure Network (V2I): This network enables a communication link between vehicles and the Road Side Unit (RSU) which will be discussed later in this session. It is established for sharing information and data gathering applications. Hybrid Network: This network combines both vehicle-to-infrastructure and vehicle-to-vehicle networks. A vehicle can establish a connection with infrastructure either directly or through vehicle-to-vehicle communication via a multi-hop connection and this is termed hybrid architecture in VANET.

VANET has special features apart from its parental MANET, which are listed below in this section. Mobility with predictable path: In VANET, the vehicles travel in a random way. Yet since the traffic rules and traffic signals are static in most of the regions, the vehicle's path is predictable. Passenger Comfort and improving efficiency of traffic: VANET allows each vehicle to communicate with other vehicles directly, which in turn reduces the delay in communication between vehicles. This type of communication enables the passenger or driver to ensure the safety regarding the roads and the vehicles traveling in the opposite direction. Point of interest (PoI) is another module in VANET which sends periodic messages about nearby gas stations, hotels and parking areas in order to offer sophisticated services to drivers.

Unconstrained Power: Unlike MANET, VANET does not have any power constraint due to the negligible power consumption of OBU for comparing the battery power of each vehicle. Oscillated Network Density: Based on the velocity of vehicles and due to traffic signals, there exists an oscillated density of vehicles in roads. Computational Capability: The vehicles are the actual nodes that are intended to communicate with each other. Since the nodes are equipped with sufficient number of sensors, AU, GPS and OBU, the computational efficiency is increased, which affords a reliable source of communication.

Ad hoc topology: The nodes in VANET are not consistent toward a single processing unit, nor do they represent a static member of currently connected topology. This occurs since an unexpected change may happen to the predefined path of any vehicle due to its commitments or due to unavoidable situations. A preliminary aspect of VANET is message dissemination and response to the requests that are posted by the vehicles or infrastructure. Some of the key challenges in VANET include Fading of Signals: The presence of an obstacle between the communication paths between two vehicles causes the signal to fade even though the vehicles are in transmission range.

Limitation in allocated Bandwidth: Bandwidth management is another key issue in VANET. The absence of any central coordinator to organize all the effects of VANET causes a problem with bandwidth and channel allocation. Connectivity: Connectivity among the vehicles is a major issue in VANET. Although the connectivity can be achieved by increasing the transmission power, this degrades the throughput. Hence, achieving connectivity between vehicles without compromising quality of service 
(QoS) factors remains an important issue to be solved (Jitendra et al., 2021). Clustering: The high priority in research issues is clustering of nodes in VANET. Though many protocols address this issue, they consider only a single aspect as opposed to all other aspects.

Routing: Designing a protocol that quickly delivers a message with high PDR and achieving less jitter is a tedious task in VANET since the topology is dynamic and the nodes are not dependent on single point of contact. The architecture and characteristics of VANET are described in detail. The next section deals with open research issues in VANET (Sunil et al., 2021). Then the motivation for this research is justified. The final sessions deal with our contribution to VANET research and the organization of paper is detailed. The next section deals with the literature survey on VANET issues, its solutions and the existing challenges.

The recent development of wireless technologies provides a radio interface between V2V communication and V2I communication that are used in VANET. These technologies are incorporated to improve road safety, prevent accidents and improve the efficiency of traffic handling and to offer sophistication to users. Cellular Systems: A cellular system is the concept of reusing the frequency channel in order to complete the task (Gerla \& Tsai, 1995). The generation of a cellular system can be defined as $2 \mathrm{G}$, 2.5G, 2.75G and 3G. 2G systems are also called Global System for Mobile (GSM) (Yu \& Chong, 2003) with a data transfer maximum rate of $9.6 \mathrm{Kbps}$. Then next-generation 2.5G is the General Packet Radio Service (GPRS) (Su \& Zhang, 2007) with higher bandwidth efficiency than the previous generation GSM. It offers a bandwidth up to $170 \mathrm{Kbps}$. The next generation is Enhanced Data Rates for GSM Evolution (EDGE) with a maximum data rate of 384Kpbs (Zhang et al., 2011). EDGE has evolved into 3G, High-Speed Downlink Packet Access (HSDPA) with a frequency of 2Mbps.

WLAN/Wi-Fi: This wireless local area network or wireless fidelity provides the communication link between vehicle-to-vehicle and vehicle-to-infrastructure with a data rate speed of $54 \mathrm{Mbps}$. It enables a communication range of $38 \mathrm{~m}$ indoors and up to $140 \mathrm{~m}$ outdoors. WiMAX: This is an extension of Worldwide Interoperability for Microwave Access (WiMAX). It has a data rate speed of 35Mbps using MIMO and OFDM. Its maximum communication range is $15 \mathrm{Kms}$. DSRC/WAVE: This is a dedicated radio technology for VANET which holds a communication range of 300 to $1000 \mathrm{~m}$ with a data rate of $27 \mathrm{Mbps}$. It is based on IEEE $802.11 \mathrm{p}$ with lower overhead operation DSRC spectrum (Ucar et al., 2013).

Clustering of nodes in VANET is one of the most recent research areas where a highly flexible clustering algorithm is required that can adapt to the dynamic changes in VANET. Although existing protocols cluster the nodes in the given region, minimizing the number of clusters is another tedious task where the spatial lattice is higher in any given VANET region which has fewer nodes. Hence, the clustering of vehicles to disseminate the information among the vehicles and the evolution of bio-inspired algorithms to obtain an optimal solution motivated the authors of this paper to develop a confronted idea and to impose a bio-inspired solution for clustering concept in VANET. Existing systems are designed for limited features which could not able to address the ad hoc nature of vehicle intelligent communication. In order to fulfill the research gap identified in VANET, a novel algorithm support is required to perform a safe and trustworthy elucidation. Data dissemination is another notable issue which need to be addressable by optimization techniques. 


\section{Literature study}

VANET facilitates communication between vehicles to avoid accidents and to support other safety measures. Data dissemination in VANET is a challenging task due to the volatile network topology. Clustering is an effective data dissemination technique with less energy consumption and lower routing overhead. However, when a vehicle leaves the cluster then it is off the shore, and therefore, the data may be lost or the lifetime of the packets expired. Hence, achieving high packet delivery ratio with lower overhead is a challenging task in VANET. This section discusses the research on VANET in terms of clustering, data dissemination and achieving high PDR. Choosing a cluster head is another recent issue that is examined in this literature survey.

The cluster head is determined using metrics like mobility of the node, unique id, displacement threshold value and signal strength, where lowest value node is elected as the cluster head (Gerla \& Tsai, 1995). Node movement and replacement are carried out when the cluster node moves out of range with each other and other mobile nodes in the cluster cannot access the cluster head (Yu \& Chong, 2003). Clustering mechanics are based on single-hop and multi-hop algorithms. Single-hop clustering in the vehicles traveling in the same direction is grouped under a single cluster ( $\mathrm{Su} \&$ Zhang, 2007). For multi-hop clustering, packet delivery delay is used to determine the cluster head which provides synchronization among nodes (Chauhan, 2021; Zhang et al., 2011).

Ucar et al. (2013) proposed a stable clustering algorithm for VANET. The authors constructed a stable clustering algorithm to share the mobility of the vehicles so as to reduce the communication overhead among the vehicles. They proposed the VMaSC Vehicular Multi-hop algorithm to provide stable clustering among vehicles. The cluster head is selected based on the lowest relative mobility calculated from the average speed of the neighbor vehicles in multiple hops. Taleb et al. (2010) proposed using the clustering-based approach in VANET for collision avoidance and a risk-aware clustering algorithm based on the metrics like velocity, direction of the vehicle and distance. To provide risk awareness, a medium access control protocol is used which helps in providing response in the cluster. The clustering is based on the movement of the direction and IEEE 802.11p. However, the vehicle velocity is inconsistent and imprudent in nature, so addition of the UMTS signal strength increases the cluster's stability.

Benslimane et al. (2011) combined VANET with 3GPP networks UMTS. In their proposed architecture, nodes are clustered dynamically based on the metrics. The issues related to 3GPP networks like gateway discovery, gateway selection and gateway processing are accomplished with the help of an adaptive mobile gateway management technique.

Souza, Evandro et al. (2010) proposed an aggregate local mobility clustering algorithm to prolong the lifetime and stability of the cluster using a beacon-based approach. This local aggregate approach re-organizes the cluster based on the contention method of selecting two cluster heads for a short time period and aggregates the local mobility vehicles to determine the cluster head that needs to be retained until another cluster head is formed.

Fan et al. (2005) proposed cluster formation using a utility-based approach. The utility functions are closest position and closest velocity. The cluster head is selected based on the utility functions, and the vehicle with the highest utility value is designated as the cluster head. However, this approach requires added functions like weighted parameters to handle dynamic traffic.

Maslekar et al. (2011) proposed a stable clustering algorithm to improve the network's efficiency so as to reduce the network traffic flow and monitor the road 
conditions. As determining the stable node in VANET is difficult, they proposed a direction-based clustering algorithm. They select the cluster head based on a switching mechanism so as to astound of clusters within the network. This methodology increases the stability of the cluster head through the switching mechanism by calculating the density value.

Wang et al. (2008) proposed a position-based clustering algorithm in which the cluster head is selected based on the geographic position of the vehicle and priorities associated within the vehicles. The priority of the vehicle is set based on their hash function. Travel time is used to calculate the hash function as thus set the priority for vehicles. The cluster head is selected based on the hash function with highest trip time. Nevertheless, this approach fails during high network traffic flows and failed to maintain the cluster head selection due to instability among the vehicles.

Wolny et al. (2008) proposed a modified distributed and mobility adaptive clustering algorithm for VANET that adapts the environment according to the mobility of the vehicles by assigning to each vehicle a weighted value based on the metrics like connectivity, mobility, position and velocity. The vehicle with highest value is considered to be the cluster head and this process avoids reelection of the cluster head, but it does increase the network's overhead.

Rasool Azimi et al. (2017) proposed a distributed data clustering algorithm for P2P networks by designing a Hybridization of centralized clustering method and gossip-based protocol approach. The proposed algorithm adapts the dynamic situation of the network and provides data clustering with a distributed approach among neighbor nodes. This system works as a central server and provides the network's stability.

Gokce Hacioglu et al. (2016) proposed a routing algorithm based on clustering in a wireless sensor network. For their clustering approach, they used a multi objective optimization algorithm non-dominated sorting genetic algorithm. To achieve a multi objective approach they used seven objective functions and the main objective is to minimize the communication cost, for which cluster selection and cluster head selection are considered. Their solutions use a different network topology and simulate sink node accordingly based on a non-dominated sorting genetic algorithm.

Zaher Merhi et al. (2015) proposed clustering among the network based on adaptive resonance theory which creates clusters in the network to provide scalability and latency effect. The clusters are created based on the speed using an unsupervised neural network based on the discrepancies of the speed. The mini clusters are based in the communication range and the cluster head is selected with the vehicle having highest number or connected vehicles. This approach provides decreased bandwidth, dynamic and mobility nature of the vehicle. Prasad Bhosale et al. (2016) proposed dynamic clustering in VANET that elects the cluster head based on the QoS parameters, which are the direction of the vehicle, speed, packet delivery ratio, transmission range, density and duration of the cluster head in their past cluster. This method is based on the agglomerate method which improves the QoS parameters in VANET.

Vijay Sharma et al. (2016) proposed a clustering approach based on density, motion ability and neighbor vehicle selection. For selecting the neighborhood selection and providing secure communication, the authors used the AES technique.

Jalalvandi et al. (2016) proposed clustering-based routing in VANET to improve the network's performance. This algorithm is scalable, distributed, dynamic and efficient. The cluster head is selected based on the low speed deviation of the vehicle, time to travel and density of the vehicle. This algorithm shows decreased end-to-end delay in the simulation result with CBLR algorithm. 
Marina Aguilera Leal et al. (2010) solved the data dissemination problem in VANET due to dynamic changes in the network topology. The traffic and collisions in the network lead to a data dissemination problem in VANET. Hence, collision might occur in another time to distribute the messages with varying densities in the network.

Niu Jianwen et al. (2009) proposed an opportunistic auto adaptive dissemination algorithm in VANET due to the stable network topology. However, the unstable communication link data prevent dissemination from exploiting the peculiarities of VANET. In this algorithm, the direction of the vehicle and number of messages are controlled to reduce the network traffic, network load and overhead caused in the cluster. This approach regulates the entry of vehicles into the network border.

Gustavo Marfia et al. (2007) proposed the tracing of a vehicular route based on the AODV algorithm and an opportunistic approach is used for routing. The authors proposed opportunistic infrastructure to improve the efficiency of the VANET communications.

DJ Dubois et al. (2013) proposed a method to exploit a wireless communication network in an opportunistic way by sharing the resources and opportunistically self-organizing the peer-to-peer network. In this infrastructure each node can act as an access point or client node in the wireless mode. This methodology is based on a context-aware technique to monitor traffic and control congestion in the network.

SK Dhurandher et al. (2013) proposed routing protocols in an opportunistic network with no infrastructure by considering extensions like mobility, connections, speed and partitions. Routing is a challenging issue in an opportunistic network due to connectivity problems among nodes. The infrastructure consumes the battery supply for the computation task. This methodology improves the performance of the wireless network by forwarding the data packet in wireless links.

Suyog and Suresh et al. (2013) recommended a cooperative and opportunistic transmission infrastructure for MANET. In this infrastructure, routing and forwarding packets are challenges due to channel interference and the absence of a central processor. Energy efficiency is an NP hard problem in an opportunistic network that can be solved using a distributed cooperative rate adaptation method in a wireless environment.

Oubbati, Omar Sami, et al. (2017) discuss the working strategy of UAVs in an ad hoc mode. It also explains the routing process embedded with VANET on the ground. The routing operation helps to improvise the reliability of the data delivery by solving the communication gap. The authors previously proposed UVAR Assisted VANETs routing protocol that enhances the connectivity of the vehicles with proper data routing on the ground by using UAVs. The drawback of using UVAR is its inability to fully exploit UAVs in the sky for carrying data forwarding. UAVs are used only when the network is placed in a poorly dense region. In this article, we propose an extended version of this protocol that supports two different ways of data routing: (i) delivering data packets particularly for the ground with UVAR-G and (ii) transmitting data packets in the sky using a reactive routing based on UVAR-S. The Simulation result explains the hybrid communication between vehicles and UAVs which can be perfectly matched for VANETs and the results are compared with other existing vehicle-to-vehicle (V2V) communications.

Abkenar, Forough Shirin and Akbar Ghaffarpour Rahbar et al. (2017) study the usage of Internet user's traffic demands. They examine the efficiency and cost for utilizing the bandwidth and spectrum in optical networks. An Elastic Optical Network (EON) is known as a new generation of optical networking, which has the capacity of high flexibility and scalability in the allocation of spectrum and data rate adaptation for supporting different types of traffic. The available resources in a network are allocated by using the EON in order to understand the user's demands. It generates elastic optical paths with variable bit 
rates and flexibly separates the available spectrum. This process is carried according to the users' traffic demands and effectively analyzes the Routing and Spectrum Allocation (RSA). Further, RSA chooses the perfect resources under the resource conditions and confirms the flexibility of the connection demands. RSA can be classified into two categories: 1) without changing format, known as simple RSA and 2) with adjustment of the modulation format, known as routing, modulation and spectrum allocation (RMSA). In this article, the proposed RSA and RMSA algorithm is incorporated for analyzing EON and the results are compared in order to identify the quality and computational accuracy.

Lee et al. (2017) study the node degree based on deep learning of wireless communication during a disaster. The virtual routes are declared according to the predecided node degree. The proposed routing method is mapped with base stations for organizing the perfect infrastructure. The authors claimed that this process can identify an effective route for assigning, conserved and regaining. The deep learning procedure is incorporated for effective classification of wireless degree nodes. The Viterbi algorithm is used for assigning virtual routes. The proposed routing method is compared with traditional methods and the results show that the proposed method surpasses the results of other traditional methods, by providing better results via simulations.

Shi, Junling, et al. (2017) propose a social-based routing scheme for a fixed-line VANET to enable the efficient and effective message routing among passengers who are separated into different communities. This separation process is completely dependent upon the Improved K -Clique community detection algorithm (IKC). The aim of the algorithm is to forward and remove the order of messages. A social-based message buffering scheme at vehicles (SMB) is introduced with their nearness and input of messages. A bilateral forwarder determination method (BFD) is proposed to understand the nature of the messages and to help to carry some functionality like forwarding, addition of Intra-Community Forwarder Determination (ICFD) and Er-Community Forwarder Determination (ECFD). The simulation results demonstrate that the proposed scheme produces better message delivery ratio and lower network overhead than other traditional methods do.

Xiu et al. (2017) study the QoS-constrained multicast routing problem. This issue is considered an NP-complete problem with varieties of swarm intelligence algorithms that fit better than other old-fashioned algorithms. A microartificial bee colony (MABC) algorithm is introduced in order to solve the quality issues. It helps to lengthen the network lifetime by reducing the delay cost. Multicast routing is converted to a continuous optimization problem and is mapped with MABC. To execute the results, it is deployed on a traffic scenario with three case studies. The optimal routes are easily obtained by using the MABC and it can be easily adopted in real time because the network design does not change too frequently.

Oliveira, Renê et al. (2017) discuss the non-trivial scientific issues that occur in VANETs, and contain the topology of an adaptive broadcast protocol. An efficient endto-end reliable warning messages dissemination is carried by the protocol. The general procedure of message communication in VANET is done by using beacon messages. This strategy formulates the details about the neighborhood information. The broadcast problem arises when the vehicles are next to each other while simultaneously broadcasting messages. This issue leads to frequent contention and broadcast-based troubles. In a sparsely populated region, vehicles face some complex issues that may reduce the network's quality such as failures in the message delivery. In this article, we propose a novel protocol, termed the adaptive data dissemination protocol (ADDP) that provides reliable message dissemination with different mechanisms in order to reduce the beacon periodicity and number of messages in the network. The performance of the protocol is mainly used to have a VANET 
safety application and the evaluation is carried through experiments with network and traffic simulators.

Khaled et al. (2017) demonstrate issues belonging to routing protocols, such as the warning messages to delivery vehicles such as issues of non-line of sight (NLOS). These situations cause erroneous message communication between the vehicles and channel contention, especially in densely populated environments. This protocol design controls the issue created by NLOS by broadcasting warning messages from emergency vehicles to vehicles and helps to reduce the overhead by enhancing the packet delivery ratio. The time delay and channel utilization are minimized by developing a routing protocol named the cooperative volunteer protocol (CVP) that can act as volunteer vehicles, for carrying the disseminate process the warning message from the source to the target vehicle understanding an NLOS situation. A novel design has been adopted by utilizing the knowledge of a context-aware system (CAS). It helps to understand the components of the OBU and their communication. It gathers data in order to make decisions based on the sensed environments. The simulation output is displayed by demonstrating that the proposed protocol outperformed the existing protocols, as measured by various metrics such as packet delivery ratio, neighborhood awareness, channel utilization, overhead and latency. The results indicate that the proposed CVP can easily detect NLOS situations. It has the capacity to solve the issues effectively and efficiently for both the intersection scenario in urban areas and the highway scenario.

Song, Chao et al. (2017) explain the different sorts of Routing in vehicular ad hoc networks (VANETs). This type of routing is dependent on the vehicle-to-vehicle communications. It faces some complex issues such as high probability and long duration among vehicles. These factors should be considered key factors while designing any routing-based network.

Chandramohan et al. (2020) propose an efficient water dropping algorithm for VANET to find an alternative root for effective and pollution-less transportation. $\mathrm{Xu} \mathrm{Bao}$ et al. (2020) applied PSO-based clustering for routing V2V for improved optimized roots efficiency. Geoffrey Eappen et al. (2020) described a Hybrid PSO-GSA for energy efficient spectrum sensing in a cognitive radio network for effective physical wireless communication. Qing Hu et al. (2020) developed a novel carrier frequency offset algorithm based on a double Barker code in VDE-TER.

Mamoudou Sangare et al. (2020) showcased and explored a forecasting approach for road accidents with analytical measures using a hybrid machine learning technique. The authors combine the descriptive strength mixture model with the high-performance classifier. It is applicable in all expert systems and their related applications. Amir Seyyedabbasi et al. (2020) described an efficient protocol for multi-agent path finding using a routing protocol based on ant colony optimization. The approach is tested in a real-time wireless sensor network and in decentralized IoT systems, with potential applications in many microprocessor-based devices.

M. F. Khan et al. (2019) proposed a clustering technique for enhanced Internet of Vehicle (IoV) by using a multi-object PSO technique and a nature-inspired algorithm to frame the clustering process. R. G. Babukarthik et al. (2020) designed a predictive mechanism for viral infection such as COVID-19 with the help of genetic deep learning convolutional neural network (GDCNN). It is supported by the PSO approach for optimized prediction. H. Khelifi et al. (2020) designed a named data networking (NDN) to drive the vehicular ad hoc networks for routing and the security mechanism for enhanced communication. D.C et al. (2020) discussed a data dissemination approach for green VANET to increase pollution-less road transportation with hybrid technique. Classifying skin disease and progress 
expectation of nominal computational efforts and advance of accurate prediction is discussed by J. Tamang et al.(2021).

\section{Cooperative behavior of VANET (CVANET)}

Vehicular ad hoc networks consist of various phases to facilitate the user and to communicate the emergency messages among vehicles. For transmitting the data among the vehicles in CVANET, several protocols and communication procedures such as clustering and routing have been proposed by many researchers to improve the robustness and reliability.

In this research work, we addressed the problem of clustering the vehicles in CVANET and developed an efficient communication procedure to address the clustering and routing process. Choosing a better cluster head significantly reduces energy consumption and improves the communication path among the vehicles to redirect the messages better and sooner. In our procedure, we imposed an improved version of a bio-inspired algorithm, namely the binary social spider algorithm, which works as a colony to achieve the global minimum points. The cooperative behavior of social spider algorithm (CBSPA) motivated the researchers to work on this algorithm to achieve improved results. The clustering and routing process of CVANET is discussed, the social spider algorithm is reviewed in detail and, finally, the mapping of social spider algorithm on CVANET is discussed for better understanding of the remaining paper structure.

\subsection{Vehicular ad hoc network}

CVANET offers enormous and significant advantages for users to avoid hazardous situations. As the usage is increased exponentially, the issues and challenges also increase. Many issues or challenges such as security-related issues, communication issues and topological issues arise as the applicability of this network increases. In this paper, the authors have addressed the clustering and reporting issue as a primary concern to improvise the data delivery throughput.

\subsection{Clustering in CVANET}

Clustering is the process of grouping a set of vehicles based on some similarity or by which one vehicle is covered by another. In VANET, clustering is difficult because the vehicles in VANET are dynamic. In our procedure, we clustered the vehicles after choosing the cluster head. Let us consider a graph $\mathrm{G}$ which consists of a vehicle $\mathrm{V}$ with its own communication range and covering range. It moves with a velocity $\mathrm{V}$

$$
\mathrm{V}=\left[\begin{array}{l}
\alpha \\
\beta \\
\gamma
\end{array}\right]
$$


where in Eq. 1, $\alpha$ represents the geographical coordination of the vehicle $\mathrm{V}, \beta$ the time at which the geographical coordination of the vehicle $\mathrm{V}$ has been recorded and $\gamma$ the velocity of vehicle $\mathrm{V}$ with respect to time $\beta$. In vehicle $\mathrm{V}, \alpha$ holds the geographical location of the vehicle in two-dimensional coordination. Hence, for each vehicle $\mathrm{V} \alpha$ can be represented as a $2 \mathrm{D}$ array as

$$
\alpha=\left[\begin{array}{l}
\alpha_{\mathrm{x}} \\
\alpha_{\mathrm{y}}
\end{array}\right]
$$

where in Eq. 2, $\mathrm{x}$, y represent the $\mathrm{x}$ and $\mathrm{y}$ coordinates of the vehicle geographical location and in terms of latitude and longitude $\mathrm{X}$ can be represented as latitude and $\mathrm{Y}$ as longitude.

\subsection{Routing in CVANET}

Routing is the process of transmitting the message from RSU to vehicles or between vehicles in an absolute path through a communication medium. Since the vehicles in roadways are not wired to each other, VANET uses a wireless medium. Routing can be achieved either through a single-hop communication in which a single point of contact is established to deliver all the messages or using multi-hop communication where a message can be transmitted to more than one vehicle to deliver it to the respective user. Another type of communication is through the cluster head by which a cluster head is chosen based on certain criteria to collect the messages from the end user or to deliver the message to the end user. In our approach, we used the cluster-based concept to deliver the messages to respective vehicles by firstly delivering them to the cluster head in each RSU range. The remaining vehicles are served through the cluster head. Hence, the communication path should be designed among the cluster heads.

\subsection{Social spider algorithm (CBSPA)}

Among the other categories, the spider social spider is a predominant category where the spiders live as a colony and behave cooperatively for their individual benefits for the benefit of the colony. Compared with other bio-inspired algorithms, social spider has the potential to work based on its gender. Based on the literature, the social spider can be segregated into two different search agents: male and female. Since a social spider colony is female biased, some females will be there in the colony when compared to male. This has been represented mathematically as

$$
\mathrm{N}_{\mathrm{f}}=\text { Floor }[(0.9-\operatorname{rand} \cdot 0.25) \cdot \mathrm{N}]
$$

where $\mathrm{N}_{\mathrm{f}}$ is the number of female population and $\mathrm{N}$ represents the size of the colony.

The male social spiders can be represented as

$$
\mathrm{N}_{\mathrm{m}}=\mathrm{N}-\mathrm{N}_{\mathrm{f}}
$$

where $\mathrm{N}_{\mathrm{m}}$ represents the number of male social spiders of colony size $\mathrm{N}$.

The weight of each spider can be represented as

$$
\mathrm{W}_{\mathrm{i}}=\frac{\text { worst }_{\mathrm{s}}-\mathrm{C}(\mathrm{s})}{\text { worst }_{\mathrm{s}}-\text { best }_{\mathrm{P}}}
$$


where $\mathrm{C}(\mathrm{s})$ is the current social spider fitness value and worst $_{\mathrm{s}}$, best $_{\mathrm{p}}$ are the worst and best fitness values of the whole colony of size N. In eqs.6, 7 and 8, the vibrations of the communal web in the social spider algorithm have been defined to identify the better solutions that are obtained during the evolution. The three types of communications between the social spiders are the vibration that is transmitted from a social spider to its nearest and the highest weight obtained spider, and it can be represented as

$$
\mathrm{Vibc}_{\mathrm{i}}=\mathrm{W}_{\mathrm{c}} \cdot \mathrm{e}^{-\mathrm{d}_{\mathrm{i}, \mathrm{x}}^{2}}
$$

The vibration that is transmitted from a social spider to the highest weight obtained spider among the whole population, and it can be represented as

$$
\operatorname{Vibb}_{\mathrm{i}}=\mathrm{W}_{\mathrm{b}} \cdot \mathrm{e}^{-\mathrm{d}_{\mathrm{i}, \mathrm{b}}^{2}}
$$

The vibration that is transmitted from a social spider to the nearest female spider and it can be represented as

$$
\operatorname{Vibf}_{\mathrm{i}}=\mathrm{W}_{\mathrm{f}} \cdot \mathrm{e}^{-\mathrm{d}_{\mathrm{i}, \mathrm{f}}^{2}}
$$

\subsection{CBSPA initialization process}

The population in the social spider algorithm is initialized for male and female social spiders separately in eqs. 9 and 10. The population initialization process for female social spiders is as follows.

$$
\mathrm{f}_{\mathrm{i}, \mathrm{j}}^{0}=\mathrm{p}_{\mathrm{j}}^{\text {low }}+\operatorname{rand}(0,1) \cdot\left(\mathrm{p}_{\mathrm{j}}^{\text {high }}-\mathrm{p}_{\mathrm{j}}^{\text {low }}\right), \mathrm{i}=1,2, \ldots, \mathrm{N}_{\mathrm{f}} ; \mathrm{j}=1,2, \ldots, \mathrm{n}
$$

where $\mathrm{p}_{\mathrm{j}}^{\text {high }}$ and $\mathrm{p}_{\mathrm{j}}^{\text {low }}$ represent the upper and lower bound of any given problem, respectively.

For male social spiders, the initialization process is.

$$
m_{k, j}^{0}=p_{j}^{\text {low }}+\operatorname{rand}(0,1) \cdot\left(p_{j}^{\text {high }}-p_{j}^{\text {low }}\right), k=1,2, \ldots, N_{m} ; j=1,2, \ldots, n
$$

\subsection{Cooperative behavior of female social spiders}

The social spider algorithm has the potential to generate new solutions based on their gender. The next-generation solution can be generated differently for female and male social spiders. In female social spiders, the next-generation population has two different strategies to search for optimal solutions based on the probability factor, and this can be represented as

$$
f_{i}^{k+1}=\left\{\begin{array}{c}
f_{i}^{k}+\alpha \cdot v i b c_{i} \cdot\left(S_{c}-f_{i}^{k}\right)+\beta \cdot v i b b_{i} \cdot\left(S_{b}-f_{i}^{k}\right)+\delta \cdot\left(\text { rand }-\frac{1}{2}\right) \text { with probability } P F \\
f_{i}^{k}+\alpha \cdot v i b c_{i} \cdot\left(S_{c}-f_{i}^{k}\right)-\beta \cdot v i b b_{i} \cdot\left(S_{b}-f_{i}^{k}\right)+\delta \cdot\left(\text { rand }-\frac{1}{2}\right) \text { with probability } 1-P F
\end{array}\right.
$$


where $f_{i}^{k+1}$ is the next-generation $(k+1)$ solution of $i$. The probability factor PF in Eq. 11 is based on the random number $(0,1)$. In male social spiders, the next-generation population has two different strategies to search for optimal solutions corresponding to the mean value of the entire male population and this can be represented as in Eq. 12,

$$
m_{i}^{k+1}=\left\{\begin{array}{c}
m_{i}^{k}+\alpha \cdot \operatorname{vibf}_{i} \cdot\left(S_{f}-m_{i}^{k}\right)+\delta \cdot\left(\operatorname{rand}-\frac{1}{2}\right) \text { if } W_{N_{f}+i}>W_{N_{f}+m} \\
m_{i}^{k}+\alpha \cdot\left(\frac{\sum_{h=1}^{N_{m}} m_{h}^{k} \cdot w_{N_{f}+h}}{\sum_{h=1}^{N_{m}} w_{N_{f}+h}}-m_{i}^{k}\right) \text { if } W_{N_{f}+i}>W_{N_{f}+m}
\end{array}\right.
$$

where $m_{i}^{k+1}$ is the next-generation $(k+1)$ solution of $i$.

\begin{tabular}{ll}
\hline Comparison of social spider algorithm and CVANET & \\
\hline SSO & CVANET \\
\hline Search space & All feasible solutions \\
Spider & A complete feasible solution \\
Population & Set of feasible solutions \\
Dimensions of a spider & Set of all vehicles \\
Female and Male Spiders & A single population set \\
Fitness & Quality of solutions \\
Best spider & Optimal combination obtained \\
Worst spider & Deviated combination from best \\
Weights of each spider & Quality of solution w.r.t best solution \\
Vibration between spiders & Quality of other solutions w.r.t neighbor solution \\
Euclidian distance between spiders & Difference between the fitness value \\
Upper and lower bound & $(011)$ \\
\hline
\end{tabular}

VANET is comprised of issues, such as clustering, routing and energy-based deployment that can be optimized to reduce the end-to-end delay and increase the throughput. Our research objective was to frame an efficient clustering and routing process that is under the optimization session. Hence, in our approach mapping, the concept of social spider algorithm would be more appropriate to the scenario of VANET is necessary. However, this section a mapping of VANET with the social spider algorithm for enhanced research clarity.

This paper deals with the existing techniques for solving cluster formation, data dissemination and the implementation of an opportunistic network in VANET. Each study presented here denotes the problem handled in their work, the algorithm they proposed, the significance of the research work, its future directions, the performance metrics they used to prove their method and the existing techniques they used for comparing their proposed research work. Finally the outcome of these research issues is taken as our objectives and it has been efficiently handled in the upcoming session.

The search space of social spider optimization (SSO) is considered to be the entire solution space where the algorithm works on it to achieve an optimal result. In VANET let us consider $U$ as the set of all feasible solutions of the VANET problem. It can be mathematically represented as $\left\{F_{1}, F_{2}, \ldots, F_{i}, \ldots, F_{m}\right\} \in U$, where $\mathrm{F}_{\mathrm{i}}$ denotes the feasible solution from $m$ number of feasible solutions.

In SSO, a spider is considered a completely feasible solution from the search space 'U.' It can be a single variable, or a one-dimensional, two-dimensional or n-dimensional arrays. 
When mapping to VANET a, a feasible solution is comprised of the vehicles inside the simulation region. In can be mathematically represented as $F_{i}=\left[V_{1}, V_{2}, \ldots, V_{p}\right]$, where $\mathrm{V}$ represents the vehicle and $p$ the number of vehicles. The population is referred to as a set of feasible solutions of a particular problem in which the solutions of the population are subject to change or altered to achieve an optimal result. In VANET, the population can be referred to as a subset of 'U.' It can be represented as Pop $\subseteq U, \operatorname{Pop}=\left\{F_{1}, F_{2}, \ldots, F_{q}\right\}$, where q represents the number of solutions in each population.

The dimensions of a spider denote the total number of decision variables that are to be optimized to yield optimal results. Concerning VANET, the dimensions denote the total number of vehicles in the simulation region. The fitness function is a problem-dependent function that obtains the fitness value of a Solution F, which can be denoted as $f(F)$.. In VANET, the quality of the solution denotes either the number of clusters formed or the energy consumed by the solution F. The best spider is considered based on the fitness value obtained $\mathrm{f}(\mathrm{F})$, corresponding to the solution F. In VANET, the best solution is represented as the set of vehicles that communicate with each other. In particular it can be represented as the vehicles participate in data transmission. It can be represented as $F^{*}=\left[V_{1}, V_{2}, \ldots, V_{p}\right]$.

The obtained solution with the lowest fitness value is considered the worst spider. With respect to VANET, the worst solution is the combination of vehicle set that gives the lowest fitness value. The weight of a spider is defined as the ratio of the difference between the worst and current solutions to the difference between the worst and best solutions. In VANET, the weight of a combination is defined in the same way. Spiders of all kinds are responsive to vibratory stimulation on their webs or on undergrowth, which alert them to the presence of prey. The Euclidian distance can be identified using the mathematical Euclidian equation between two points. In VANET, the difference between the fitness value of each combination of vehicles is taken accounted. The upper and lower bounds represent the maximum and minimum values of each decision variable, respectively. In VANET, it is defined as a binary format. If the Boolean value 1 has been represented, then the vehicle is chosen either as the cluster head or as the communication medium.

\subsection{Binary social spider algorithm for efficient clustering in CVANET}

Clustering is the process of grouping nodes to form several clusters for efficient data discrimination with minimal power consumptions and other major objectives. In VANET, vehicles are considered to be nodes and each vehicle can be served as one among the two major roles of clustering: cluster head $(\mathrm{CH})$ or cluster member $(\mathrm{CM}) . \mathrm{CH}$ is the vehicle in that particular cluster that is responsible for data dissemination and collection. Each cluster has one vehicle as its $\mathrm{CH}$ and the other remaining vehicles act as CM.

However, in VANET achieving clustering is difficult due to the characteristics of VANET such as high mobility and abrupt changes in vehicle velocity. Hence, normal clustering methods which are designed for WSN or MANET are incapable of achieving clustering in VANET. For designing a clustering process in VANET the characteristics must be considered for efficient data dissemination. A bio-inspired algorithm needs to be incorporated since clustering is an NP hard problem. This section is followed by the system model and then by the modified SSO algorithm.

Let the simulation region be $\mathrm{G}$ which consists of $\mathrm{N}$ vehicles such that $\mathrm{G}=\left\{\mathrm{V}_{1}, \mathrm{~V}_{2}, \ldots, \mathrm{V}_{\mathrm{i}}, \ldots, \mathrm{V}_{\mathrm{N}}\right\}$. Each vehicle $\mathrm{V}_{\mathrm{i}}$ in $\mathrm{G}$ consists of a spatiotemporal message which consists of the geographical region with respect to time and the velocity of the 
vehicle, which can be represented as $V_{i}=\left[\begin{array}{c}\alpha_{i} \\ \beta_{i} \\ \gamma_{i}\end{array}\right]$, where $\alpha_{i}$ represents the geographical coordination of the vehicle $V_{i}$., $\beta_{i}$. the time at which the geographical coordination of the vehicle $V_{i}$. has been recorded and $\gamma_{i}$. the velocity of vehicle $V_{i}$ with respect to time $\beta_{i}$. Each $\alpha_{i}$ in vehicle $\mathrm{V}$ holds the geographical location of the vehicle in two-dimensional coordination. Hence, for each vehicle. A can be represented as a 2D array as.

$i \in\{1,2, \ldots, N\}=\left[\begin{array}{c}\alpha_{\mathrm{xi}} \\ \alpha_{\mathrm{yi}}\end{array}\right]$, where $\mathrm{x}, \mathrm{y}$ represent the $\mathrm{x}$ and $\mathrm{y}$ coordinates of the vehicle geographical $\mathrm{V}_{\mathrm{i}}$ location and $\mathrm{x}$ can be represented as latitude and $\mathrm{y}$ as longitude.

Observation 1 Let the given $\mathrm{G}$ be a simulation region that is fixed with the roads within its boundary the form of 2 lanes. Each pair of lanes on the road runs in an opposite direction and the vehicles in the lane are traveling in the same lane as it is initiated.

In Fig. 1, the vehicles in the simulation region $G$ can be grouped into two different sets $V_{L}$ and $V_{R}$ that represent the set of vehicles that travel in the left and right lanes, respectively. This can be represented as $V_{L}=\left\{V_{1}, V_{2}, \ldots, V_{i}, \ldots, V_{\left|V_{L}\right|}|| V_{L}|\leq| V \mid\right\}$ and $V_{R}=V_{L}^{C}$, where $C$ represents the complement of $V_{L}$ and II represent the cardinality. This can be written as $V=V_{L} \cup V_{R}$ such that $\left(\forall V_{L}, V_{R} \in V\right) V_{L} \neq V_{R} \Rightarrow V_{L} \cap V_{R}=\emptyset$.

Observation 2 Let us consider that the clusters are formed among the vehicles that travel in the same lane with respect to their velocity. For the instances of time $\mathrm{T}_{1}$ and $\mathrm{T}_{2}$, let us compute the distance between two vehicles such that in eqs.12 and 13,

$$
\begin{aligned}
& D T_{1}=\sqrt{\left.\left.\left(\left(V_{j}\left(\alpha_{\mathrm{xj}}\right)\right)_{T_{1}}-\left(V_{i}\left(\alpha_{\mathrm{xi}}\right)\right)_{T_{1}}\right)\right)^{2}+\left(\left(V_{j}\left(\alpha_{\mathrm{yj}}\right)\right)_{T_{1}}-\left(V_{i}\left(\alpha_{\mathrm{yi}}\right)\right)_{T_{1}}\right)\right)^{2}} \\
& D T_{T_{2}}=\sqrt{\left.\left.\left(\left(V_{j}\left(\alpha_{x j}\right)\right)_{T_{2}}-\left(V_{i}\left(\alpha_{x i}\right)\right)_{T_{2}}\right)\right)^{2}+\left(\left(V_{j}\left(\alpha_{y i}\right)\right)_{T_{2}}-\left(V_{i}\left(\alpha_{y i}\right)\right)_{T_{2}}\right)\right)^{2}}
\end{aligned}
$$

Fig. 1 VANET clustering concept

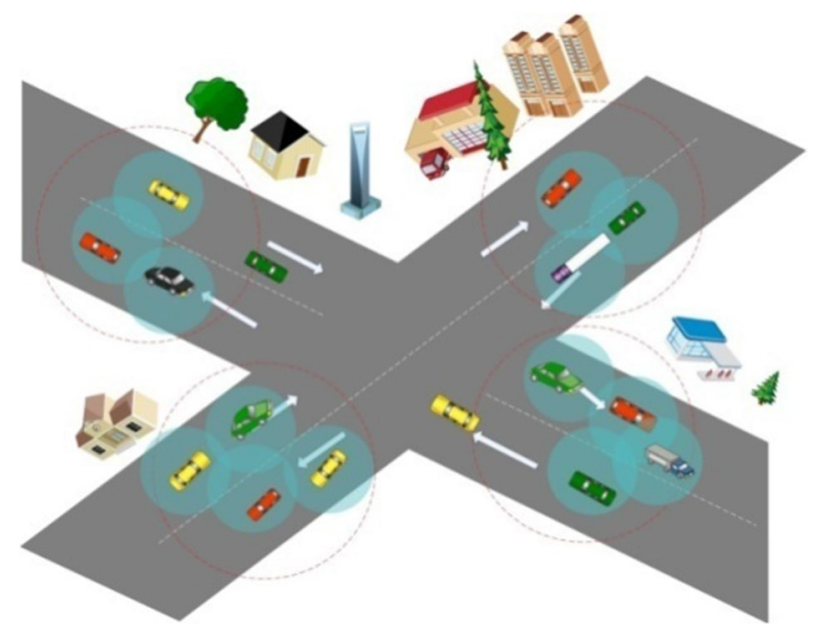


where $V_{I}$ and $V_{j}$ are the vehicles of given simulated region $G$. From this above-given equation, we can obtain when $\mathrm{T}_{1} \leq \mathrm{T}_{2}$ and $\mathrm{DT}_{\mathrm{T}_{1}} \geq \mathrm{DT}_{\mathrm{T}_{2}}$ then vehicles $I$ and $\mathrm{V}_{\mathrm{j}}$ flow in the same direction or in the same lane. When $\mathrm{T}_{1} \leq \mathrm{T}_{2}$ and $\mathrm{DT}_{\mathrm{T}_{1}}<\mathrm{DT}_{\mathrm{T}_{2}}$ then vehicles $\mathrm{I}_{\mathrm{i}}$ and $\mathrm{V}_{\mathrm{j}}$ flow in opposite directions or in different lanes.

Observation 3 In this objective, the total number of clusters in the simulation region $\mathrm{G}$ is defined as the fitness value. With respect to the social spider algorithm, the distance between the solutions is defined as the total energy consumed by the clusters in order to communicate with the cluster head in Eq. 14. It can be formulated as where, $\sum_{i=1}^{T_{C H}} \sum_{j=1}^{\left|C H_{i}\right|} \mathrm{CH}_{i} \times d_{i, j}$

$$
d_{i, j}=\sqrt{\left(\left(C H_{i}\left(\alpha_{x i}\right)\right)-\left(C H_{i, j}\left(\alpha_{x i, j}\right)\right)^{2}+\left(C H_{i}\left(\alpha_{y i}\right)\right)-\left(C H_{i, j}\left(\alpha_{y i, j}\right)\right)^{2}\right)}
$$

such that $\mathrm{CH}_{i} \in(0,1)$.

\section{Modified social spider algorithm for efficient clustering in CVANET}

SSO is an optimization algorithm inspired by the cooperative nature between spiders in the colony. Since the solution space of spider insects is continuous in nature the algorithm has been derived in a form capable of efficiently solving the continuous space problems. However, since it is an evolutionary process algorithm, many problems evolve toward optimal solution if it gets tuned. The social spider mimics not only the evolution using the cooperative behavior of spiders but also possesses a special quality of evolution which is the bisexual mating quality of the species. This avoids the premature convergence of solutions which leads toward optimal solutions in an iterative manner.

In our algorithm and in the procedure for solving the clustering process in CVANET using SSO, we incorporated significant changes to the conventional SSO algorithm for efficiently solving the clustering problem in CVANET. The changes are described in these sections which are reflected in the SSO algorithm which is then termed the modified SSO algorithm (M-SSO), which consists of the following changes in initialization process, solution repair and modification in modeling of vibrations through communal web, adaptive cooperative behavior in female spider and also in male spider cooperative behavior. These four features adapt the binary representation of any problem which can be solved through SSO.

\subsection{Binary individual representation}

An individual in an evolutionary algorithm represents a complete feasible solution which has the tendency to evolve toward the optimal feasible solution due to the sarcastic change in the individuals during evolution with the help of operators. In the conventional SSO algorithm, the solution representation is continuous variables since the search space is continuous in nature. In this clustering module in CVANET, the nodes in the region either have to be chosen as a $\mathrm{CH}$ or $\mathrm{CM}$. This allows a solution to be found in the binary format of Eq. 15. In our proposed M-SSO, the solutions are represented in the form of Boolean values. Hence, the proposed initialization format can be mathematically formulated as 


$$
\left(\operatorname{Indv}_{\mathrm{i}} \mathrm{i} \in 1,2 \ldots,|\mathrm{Pop}|\right)=\left[\mathrm{V}_{\mathrm{i}, 1}, \mathrm{~V}_{\mathrm{i}, 2}, \ldots, \mathrm{V}_{\mathrm{i}, \mathrm{j}}, \ldots \mathrm{V}_{\mathrm{i}, \mathrm{N}}\right] \mid \mathrm{j} \in \mathrm{V}
$$

An individual consists of all the vehicles in the region $\mathrm{G}$ for choosing the cluster heads among it.Indv $=\left[\mathrm{V}_{\mathrm{L}} \cup \mathrm{V}_{\mathrm{R}}\right]$. In Eq. 16, each $\mathrm{V}$ of Indv holds the values 0 and 1, $\mathrm{V}_{\mathrm{i}, \mathrm{j}} \in 0,1|\mathrm{i} \in| \mathrm{Pop} \mid, \mathrm{j} \in \mathrm{V}$ When the Vehicle $\mathrm{V}_{\mathrm{i}, \mathrm{j}}$ represents 1 , then it has been chosen as $\mathrm{CH}$; otherwise, it goes to $\mathrm{CM}$ of any cluster and it can be mathematically formulated as

$$
V_{i, j}=\left\{\begin{array}{lc}
\mathrm{CH} & V_{i, j}=1 \\
\mathrm{CM} & \text { otherwise }
\end{array}\right.
$$

After the initialization of all individuals of the iteration, the solutions should be resolved in terms of a solution-repairing process. Each individual generated from the binary individual representation consists of a random set of Boolean values. When the chosen vehicle which acts as a cluster head belongs to the same cluster (i.e. when the chosen vehicles are in same transmission range of vehicles), then $\mathrm{CH}$ has to be reduced to a single one among the chosen. After initialization, the vehicles in an individual can be grouped into $\mathrm{CH}$ nodes and $\mathrm{CM}$ nodes. The vehicles which are chosen as the cluster head can be represented in Eq. 17, as $\mathrm{CH}_{\mathrm{i}}=\left\{\mathrm{V}_{\mathrm{i}, \mathrm{j}} \in 1 \mid \mathrm{i} \in \mathrm{Indv}, \mathrm{j} \in \mathrm{V}\right\}$ and the cluster members of each vehicle in $\mathrm{CH}$ set can be represented as

$$
\mathrm{CM}_{\mathrm{CH}_{i}}=\left\{V_{i, j} d_{i, j} \leq \text { Trans Range, } i \in \mathrm{CH}, j \in 1 \ldots N\right\}
$$

After the segregation of the individual into $\mathrm{CH}$ and $\mathrm{CM}$ subsets such that Indv $=\mathrm{CH} \cup \mathrm{CM}$, the vehicles belonging to $\mathrm{CM}$ of each $\mathrm{CH}$ are cross-checked to determine whether any one of the vehicles lies in both the sets, in which case the element in $\mathrm{CH}$ is redundant and is removed. And the $\mathrm{CH}$ array is restructured by removing an element which lies in CM. The SSO algorithm possesses a cooperative nature among the colony members. The cooperative behavior exists in this SSO algorithm for effective message signaling about the food source or the attraction toward its neighboring mates in Eq. 18. In the conventional SSO algorithm the modeled attraction behavior for each individual that belongs to the female community is purely built as per their solution space. Since their solution space is continuous in nature, the same phenomena have been incorporated. Since our model requires a binary representation, the updating behavior of female spiders is modified as

$$
\operatorname{FIndv}_{\mathrm{i}}^{\mathrm{t}+1}=\left\{\begin{array}{l}
\operatorname{FIndv}_{\mathrm{i}}^{\mathrm{t}} \vee \mathrm{V}_{\mathrm{ibci}} \cdot\left(\operatorname{Indv}_{\mathrm{c}} \wedge \operatorname{FIndv}_{\mathrm{i}}^{\mathrm{t}}\right) \vee \mathrm{V}_{\mathrm{ibbi}} \cdot\left(\operatorname{Indv}_{\mathrm{b}} \wedge \operatorname{FIndv}_{\mathrm{i}}^{\mathrm{t}}\right) \mathrm{r}_{1}<\gamma \\
\operatorname{FIndv}_{\mathrm{i}}^{\mathrm{t}} \wedge \mathrm{V}_{\mathrm{ibci}} \cdot\left(\operatorname{Indv}_{\mathrm{c}} \vee \operatorname{FIndv}_{\mathrm{i}}^{\mathrm{t}}\right) \wedge \mathrm{V}_{\mathrm{ibbi}} \cdot\left(\operatorname{Indv}_{\mathrm{b}} \vee \operatorname{FIndv}_{\mathrm{i}}^{\mathrm{t}}\right) \mathrm{r}_{\mathrm{l}} \geq \gamma
\end{array}\right.
$$

where $\wedge$ represents logical AND operator and $\vee$ represents logical OR operator. The vibration occurrence through communal web can be reflected to the above-mentioned equation in the form of eqs. 19 and 20.

$$
\mathrm{V}_{\mathrm{ibci}}=\left\{\begin{array}{cc}
1^{\prime s} C(.) & \mathrm{V}_{\mathrm{ibci}}>\mathrm{V}_{\mathrm{ibbi}} \\
2^{\prime s} C(.) & \text { otherwise }
\end{array}\right.
$$

and

$$
\mathrm{V}_{\mathrm{ibbi}}=\left\{\begin{array}{cc}
1^{\prime \mathrm{s}} C(.) & \mathrm{V}_{\mathrm{ibbi}}>\mathrm{V}_{\mathrm{ibci}} \\
2^{\prime \mathrm{s}} C(.) & \text { otherwise }
\end{array}\right.
$$


In Eqs. 19 and 20, $1^{\prime} \mathrm{s}$ (.) denotes 1's complement of the given binary sector and $2^{\prime}$ (.) denotes 2's complement. In this section, the binary SSO algorithm pseudocode has been developed to solve the clustering problem in CVANET. The working process of B-SSO is followed based on this pseudocode. Data dissemination among the clusters requires a special computation in order to reduce the overall transmission cost and efficient routing model. The next hop to be communicated will be defined and chosen as per the requirement. New cluster identified to the respective clusters based on this proposed methodology. The solution repair of B-SSO has been redefined based on the requirements required. Hence, each individual in this module will consists of only the cluster head vehicles and this can be represented as

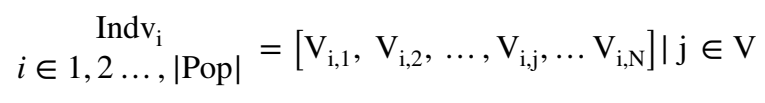

In a simulation region, no vehicle can communicate with each other unless there exists a link between any two vehicles or any two cluster head nodes by Eq. 21 . Hence, the neighbor nodes from the chosen cluster heads have to be arranged such that there exists a link between those two neighbor clusters. Hence, in this system model apart from the system model and assumptions which are depicted from issues identified, the individual representation should be done such that each node should be within its neighborhood transmission range. Choose a node $\mathrm{j}$ which can be a neighbor of node $\mathrm{i}$ such that the distance is less than or equal to twice of the transmission range shown in Eq. 22. This can be mathematically formulated as

$$
\text { Indv }_{\mathrm{i}+1}^{\mathrm{z}}=\mathrm{V}_{\mathrm{j}} \mid \mathrm{d}(\mathrm{i}, \mathrm{j}) \leq 2(\text { TransRange }) \forall \mathrm{j} \in \mathrm{V}_{\mathrm{NP}}, \mathrm{CM}_{\mathrm{i}, \mathrm{k}} \neq \mathrm{CM}_{\mathrm{j}, \mathrm{k}}
$$

where $\mathrm{z}$ represents the individual and $\mathrm{i}$ represents the position of present vehicle in individual $\mathrm{z}$ and $\mathrm{i}+1$ represents the neighbor position of node $i, d(i, j)$ is the distance between node $\mathrm{i}$ and node $\mathrm{j}, \mathrm{CM}$ denotes the cluster members and $\mathrm{k}$ represents the cluster. New cluster is illustrated in Eqs. 23 and 24, and if the $\mathrm{CM}_{\mathrm{j}}$ of any cluster has been chosen to be the neighborhood vehicle of vehicle $i$, then the next node should be the $\mathrm{CH}$ of node $\mathrm{j}$ should be placed as neighborhood of node $\mathrm{j}$. This can be mathematically represented as

$$
\operatorname{Indv}_{\mathrm{i}+1}^{\mathrm{z}}=\mathrm{CH}_{\mathrm{i}}^{\mathrm{z}} \text { if }\left(\operatorname{Indv}_{\mathrm{i}}^{\mathrm{z}} \in \mathrm{CH}_{\mathrm{i}}^{\mathrm{z}} \& \mathrm{CH}_{\mathrm{i}}^{\mathrm{z}} \notin \mathrm{V}_{\mathrm{NP}}\right)
$$

The above-mentioned equations can be clubbed and written as

$$
\operatorname{Indv}_{\mathrm{i}+1}^{\mathrm{z}}=\left\{\begin{array}{c}
\mathrm{CH}_{\mathrm{i}}^{\mathrm{z}} \operatorname{Indv}_{\mathrm{i}}^{\mathrm{z}} \in \mathrm{CH}_{\mathrm{i}}^{\mathrm{z}} \mathrm{CH}_{\mathrm{i}}^{\mathrm{z}} \notin \mathrm{V}_{\mathrm{NP}} \\
\mathrm{V}_{\mathrm{j}} \mid \mathrm{d}(\mathrm{i}, \mathrm{j}) \leq 2(\text { TransRange }) \forall \mathrm{j} \in \mathrm{V}_{\mathrm{NP}}, \mathrm{CM}_{\mathrm{i}, \mathrm{k}} \neq \mathrm{CM}_{\mathrm{j}, \mathrm{k}}
\end{array}\right.
$$




\subsection{M-SSO for clustering algorithm}

Step 1: Initialize G, Trans Range, PopSize, $f(), w()$, SSO parameters Step 2: Initialize the Pop based on proposed Binary Solution Representation (BSR) using Eq. (4.7) and (4.8)

end for

$$
\begin{gathered}
\forall i \in \text { Pop } \\
\text { Ind } v_{i} \leftarrow B S R(.)
\end{gathered}
$$

Step 3: Repair each Individual based on the solution repair operator using Eq. (4.9) and Eq. (4.10)

Step 4: Generate the number of male and female spider population based on Eq. (3) such that

$$
\text { Pop } \leftarrow \text { FInd } v \cup \text { MInd } v
$$

Step 5: For each spider calculate the fitness value $\forall i \in P o p$

end for

$$
f\left(\operatorname{Ind} v_{i}\right) \leftarrow T_{C H}
$$

Step 6: Calculate the weight for each spider based on their energy consumed with their CH's using Eq. 4.5 and Eq. 4.6

$\forall i \in P o p$

$w_{i} \leftarrow$ using Eq. 4.5 and 4.6 end for

Step 7: Set $t \leftarrow 1 / /$ Starting with the 1st iteration

Step 8: Repeat through Step 13Untilt $\leq$ Max_IT, else go to Step 14

Step 9: For every Individual in Female Individual population move the spiders based on proposed Cooperative behaviour.

Step 9.1: Repeat through Step 9.3Until $i \leq \mid$ FInd $v_{t} \mid$, such that $i \in$ FIndv else goto Step 10

Step 9.2: Calculate Vibrations among the communal web $\mathrm{Vib}_{b}, \mathrm{Vib}_{c}$ Step 9.3: FIndv $v_{i}^{t+1} \leftarrow M F C B\left(\right.$ FIndv $\left.v_{i}^{t}\right)$

// Position of the Individual of next generation by applying proposed MFCB using Eq. (4.11)

// After this process of cooperative behaviour among each individual in female population the main Population will be updated.

Step 10: For every Individual in Male Individual population move the spiders based on proposed Cooperative behaviour

Step 10.1: Repeat through Step 10.3Until $i \leq \mid$ MIndv $v_{t} \mid$, such that $i \in$ MIndv else goto Step 11

Step 10.2: Calculate Vibrations among the communal web Vib

Step 10.3: MIndv $v_{i}^{t+1} \leftarrow M M C B\left(M I n d v_{i}^{t}\right) \quad / /$ Position of the Individual of next generation by applying proposed $M M C B$ using Eq. (4.12)

// After this process of cooperative behaviour among each individual in male population the main Population will be updated.

Step 11: For each spider in the population calculate the fitness value

$$
\forall i \in P o p
$$

$f\left(I n d v_{i}^{t+1}\right) \leftarrow T_{C_{i}} / /$ number of clusters for new population

$$
\text { end for }
$$

Step 12: Calculate the weight for each spider based on their energy

consumed with their CH's using Eq. 4.5 and Eq. 4.6

$$
\forall i \in P \text { op }
$$

$w_{i}{ }^{t+1} \leftarrow$ using Eq. 4.5 and 4.6

end for

Step 13: $t \leftarrow t+1 / /$ increase the iteration number

Step 14: Find the best individual among the individuals of Pop ${ }^{\text {Max_IT }}$ and display the minimum number of clusters it produced for broadcasting the messages in CVANET

$$
\text { Optimal number of clusters } \leftarrow \min _{k \in\{1 \ldots \text { Popsize }\}}\left(f\left(\operatorname{Ind} v_{k}^{\text {Max } \_ \text {IT }}\right)\right.
$$


Table 1 Parameter settings

\begin{tabular}{ll}
\hline Parameters & Values \\
\hline Population size & 100 \\
Maximum iterations & 500 \\
Total runs & 10 \\
Vehicle velocity & $50-70 \mathrm{~km} / \mathrm{h}$ \\
Simulation area & $1 \times 1 \mathrm{Km}^{2}, 2 \times 2 \mathrm{Km}^{2}, 3$ \\
& $\times 3 \mathrm{Km}^{2}, 4 \times 4 \mathrm{Km}^{2}$ \\
Maximum acceleration & $90 \mathrm{~km} / \mathrm{h}$ \\
Minimum distance between vehicles & $20 \mathrm{~m}$ \\
Maximum distance between vehicles & $\mathrm{NA}$ \\
Lane width & $50 \mathrm{~m}$ \\
Total lanes & 2 \\
Transmission range & $100-600$ \\
Mobility models & Freeway Mobility Model \\
\hline
\end{tabular}

\section{Variables Used.}

$\mathrm{V} \leftarrow$ Total set of vehicles in the simulation region.

$\mathrm{G} \leftarrow$ Simulation region.

PopSize $\leftarrow$ Population size / no. of feasible solutions.

$\mathrm{V}_{\mathrm{L}} \leftarrow$ Vehicles in the Left lane.

$\mathrm{V}_{\mathrm{R}} \leftarrow$ Vehicles in the Right lane.

$\mathrm{DT}_{\mathrm{T}_{1}} \leftarrow$ Distance between two vehicles at time $\mathrm{T} 1$.

$\mathrm{DT}_{\mathrm{T}_{2}} \leftarrow$ Distance between two vehicles at time $\mathrm{T} 2$.

Pop $\leftarrow$ Possible solutions / Search space.

Indv $\leftarrow$ Single solution in the possible solutions / Search space.

$\mathrm{CH} \leftarrow$ subset of the individual which holds the cluster head nodes.

$\mathrm{CM} \leftarrow$ subset of the individual which holds the cluster members for each $\mathrm{CH}$.

$\mathrm{d}_{\mathrm{i}, \mathrm{j}} \leftarrow$ Distance between two vehicles at a given instance.

TransRange $\leftarrow$ Transmission range of each vehicle.

FIndv $\leftarrow$ subset of the pop which holds Female individuals.

MIndv $\leftarrow$ subset of the pop which holds male individuals.

$\mathrm{V}_{\text {ibci }} \leftarrow$ vibrant information from individual c.

$\mathrm{V}_{\text {ibfi }} \leftarrow$ vibrant information from individual $\mathrm{f}$.

$\mathrm{V}_{\text {ibbi }} \leftarrow$ vibrant information from individual $\mathrm{b}$.

$\mathrm{W}_{\mathrm{i}} \leftarrow$ weight of each individual.

$\mathrm{t} \leftarrow$ current iteration.

Max_IT $\leftarrow$ Maximum number of iterations

Observation 4 Transmission Range of each vehicle is the same and constant. The number of vehicles in the simulation region is constant. The distance between each $\mathrm{CM}$ and their respective $\mathrm{CH}$ is considered the total energy consumed for transmission. 
A. Shankar et al.

Table 2 Results of total number of clusters of different algorithms for grid 1X1

\begin{tabular}{|c|c|c|c|c|c|}
\hline Nodes & $\begin{array}{l}\text { Transmission } \\
\text { range }\end{array}$ & GA & CLPSO & $\mathrm{ACO}$ & M-SSO \\
\hline \multirow[t]{11}{*}{30} & 100 & 28 & 25 & 22 & 12 \\
\hline & 150 & 22 & 22 & 15 & 11 \\
\hline & 200 & 19 & 14 & 9 & 7 \\
\hline & 250 & 17 & 15 & 8 & 4 \\
\hline & 300 & 14 & 11 & 5 & 5 \\
\hline & 350 & 12 & 10 & 6 & 3 \\
\hline & 400 & 14 & 10 & 7 & 4 \\
\hline & 450 & 13 & 10 & 5 & 3 \\
\hline & 500 & 9 & 6 & 4 & 3 \\
\hline & 550 & 10 & 7 & 5 & 4 \\
\hline & 600 & 10 & 8 & 5 & 2 \\
\hline 30 & 100 & 26 & 24 & 14 & 11 \\
\hline 35 & & 28 & 26 & 15 & 17 \\
\hline 40 & & 31 & 30 & 19 & 18 \\
\hline 45 & & 35 & 31 & 22 & 23 \\
\hline 50 & & 37 & 32 & 23 & 24 \\
\hline 55 & & 38 & 36 & 24 & 23 \\
\hline 60 & & 40 & 38 & 25 & 23 \\
\hline \multirow[t]{11}{*}{40} & 100 & 30 & 28 & 18 & 15 \\
\hline & 150 & 27 & 24 & 15 & 10 \\
\hline & 200 & 22 & 19 & 9 & 6 \\
\hline & 250 & 17 & 14 & 5 & 7 \\
\hline & 300 & 14 & 10 & 6 & 3 \\
\hline & 350 & 12 & 10 & 7 & 4 \\
\hline & 400 & 13 & 8 & 5 & 3 \\
\hline & 450 & 11 & 7 & 5 & 3 \\
\hline & 500 & 10 & 6 & 4 & 2 \\
\hline & 550 & 9 & 5 & 3 & 2 \\
\hline & 600 & 9 & 6 & 3 & 1 \\
\hline 30 & 200 & 19 & 16 & 8 & 6 \\
\hline 35 & & 19 & 16 & 8 & 6 \\
\hline 40 & & 20 & 17 & 7 & 7 \\
\hline 45 & & 20 & 17 & 7 & 7 \\
\hline 50 & & 21 & 18 & 9 & 8 \\
\hline 55 & & 21 & 18 & 9 & 8 \\
\hline 60 & & 22 & 19 & 10 & 9 \\
\hline
\end{tabular}


Table 2 (continued)

\begin{tabular}{|c|c|c|c|c|c|}
\hline Nodes & $\begin{array}{l}\text { Transmission } \\
\text { range }\end{array}$ & GA & CLPSO & $\mathrm{ACO}$ & M-SSO \\
\hline \multirow[t]{11}{*}{50} & 100 & 37 & 31 & 22 & 18 \\
\hline & 150 & 33 & 23 & 15 & 13 \\
\hline & 200 & 28 & 19 & 10 & 10 \\
\hline & 250 & 21 & 14 & 7 & 5 \\
\hline & 300 & 18 & 11 & 6 & 5 \\
\hline & 350 & 15 & 9 & 5 & 3 \\
\hline & 400 & 12 & 8 & 5 & 3 \\
\hline & 450 & 13 & 9 & 3 & 5 \\
\hline & 500 & 11 & 6 & 3 & 1 \\
\hline & 550 & 8 & 5 & 2 & 1 \\
\hline & 600 & 9 & 5 & 2 & 1 \\
\hline 30 & 300 & 13 & 12 & 5 & 3 \\
\hline 35 & & 13 & 10 & 4 & 2 \\
\hline 40 & & 14 & 11 & 5 & 3 \\
\hline 45 & & 14 & 11 & 5 & 2 \\
\hline 50 & & 15 & 12 & 4 & 2 \\
\hline 55 & & 15 & 11 & 5 & 2 \\
\hline 60 & & 16 & 12 & 5 & 1 \\
\hline \multirow[t]{11}{*}{60} & 100 & 35 & 27 & 24 & 18 \\
\hline & 150 & 27 & 21 & 17 & 14 \\
\hline & 200 & 22 & 18 & 13 & 10 \\
\hline & 250 & 20 & 14 & 10 & 9 \\
\hline & 300 & 19 & 13 & 8 & 10 \\
\hline & 350 & 17 & 13 & 8 & 9 \\
\hline & 400 & 16 & 13 & 6 & 5 \\
\hline & 450 & 15 & 11 & 6 & 4 \\
\hline & 500 & 11 & 9 & 5 & 7 \\
\hline & 550 & 10 & 8 & 3 & 1 \\
\hline & 600 & 10 & 7 & 3 & 1 \\
\hline 30 & 400 & 10 & 8 & 4 & 2 \\
\hline 35 & & 10 & 8 & 4 & 1 \\
\hline 40 & & 11 & 8 & 3 & 2 \\
\hline 45 & & 11 & 9 & 3 & 1 \\
\hline 50 & & 12 & 8 & 3 & 1 \\
\hline 55 & & 12 & 9 & 2 & 1 \\
\hline 60 & & 13 & 10 & 4 & 1 \\
\hline
\end{tabular}


Fig. 2 Clusters vs varied transmission range with fixed nodes 30 of Grid 1X1

Fig. 3 Clusters vs varied transmission range with fixed nodes 40 of Grid 1X1

Fig. 4 Clusters vs varied transmission range with fixed nodes 50 of Grid $1 \mathrm{X} 1$

Fig. 5 Clusters vs varied transmission range with fixed nodes 60 of Grid 1X1
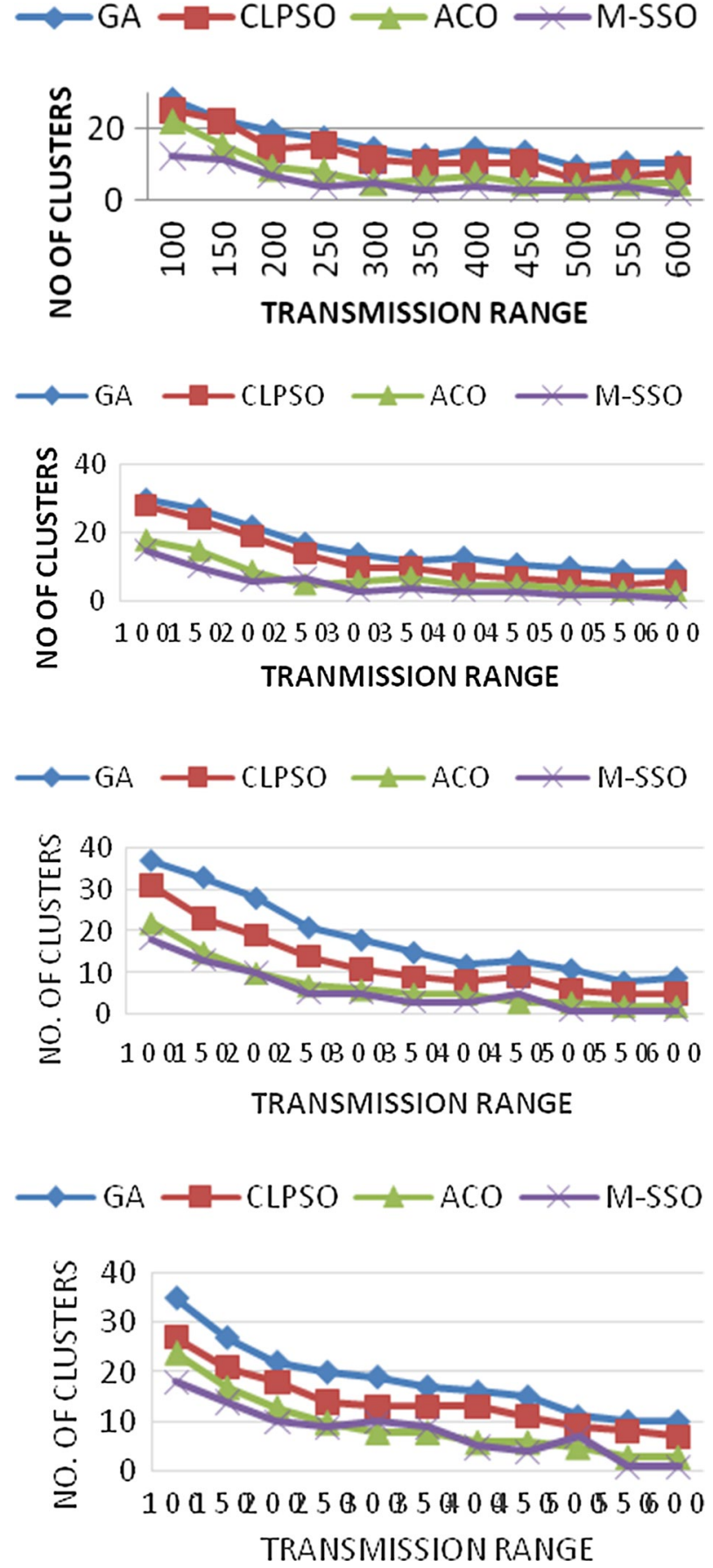
Fig. 6 Clusters vs varied \#nodes with fixed transmission range 100 of Grid $1 \mathrm{X} 1$

Fig. 7 Clusters vs varied \#nodes with fixed transmission range 200 of Grid $1 X 1$

Fig. 8 Clusters vs varied \#nodes with fixed transmission range 300 of Grid $1 X 1$

Fig. 9 Clusters vs varied \#nodes with fixed transmission range 400 of Grid 1X1
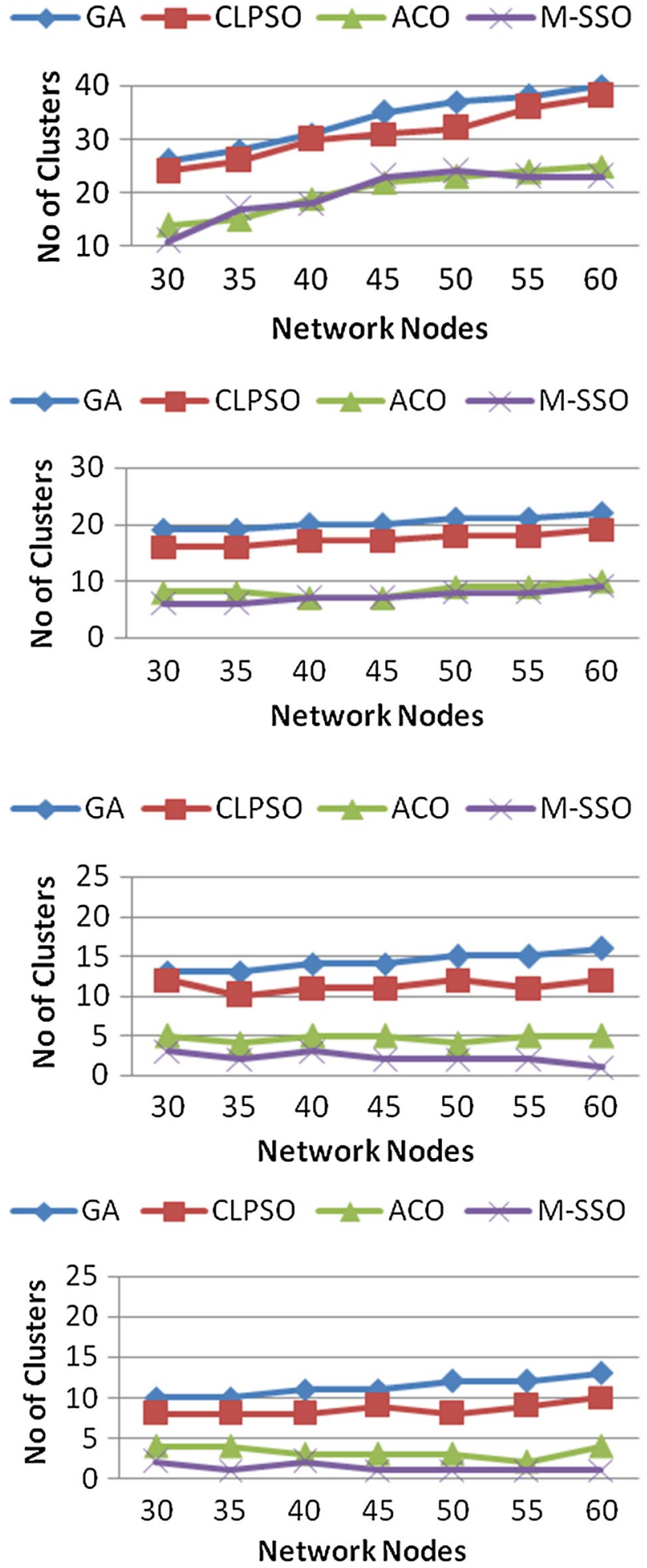
A. Shankar et al.

Table 3 Results of total number of clusters of different algorithms for grid $2 \mathrm{X} 2$

\begin{tabular}{|c|c|c|c|c|c|}
\hline Nodes & $\begin{array}{l}\text { Transmission } \\
\text { range }\end{array}$ & GA & CLPSO & $\mathrm{ACO}$ & M-SSO \\
\hline \multirow[t]{11}{*}{30} & 100 & 30 & 28 & 23 & 19 \\
\hline & 150 & 29 & 27 & 19 & 16 \\
\hline & 200 & 24 & 24 & 15 & 14 \\
\hline & 250 & 26 & 22 & 12 & 11 \\
\hline & 300 & 22 & 19 & 11 & 9 \\
\hline & 350 & 23 & 16 & 9 & 7 \\
\hline & 400 & 21 & 17 & 9 & 6 \\
\hline & 450 & 20 & 15 & 7 & 5 \\
\hline & 500 & 17 & 13 & 6 & 3 \\
\hline & 550 & 16 & 11 & 5 & 2 \\
\hline & 600 & 14 & 12 & 5 & 3 \\
\hline 30 & 100 & 21 & 19 & 11 & 10 \\
\hline 35 & & 23 & 20 & 11 & 10 \\
\hline 40 & & 24 & 22 & 12 & 11 \\
\hline 45 & & 26 & 24 & 13 & 12 \\
\hline 50 & & 27 & 25 & 14 & 13 \\
\hline 55 & & 29 & 25 & 15 & 14 \\
\hline 60 & & 30 & 26 & 17 & 15 \\
\hline \multirow[t]{11}{*}{40} & 100 & 42 & 37 & 29 & 25 \\
\hline & 150 & 39 & 40 & 25 & 19 \\
\hline & 200 & 40 & 33 & 20 & 16 \\
\hline & 250 & 34 & 27 & 16 & 18 \\
\hline & 300 & 27 & 23 & 14 & 11 \\
\hline & 350 & 28 & 20 & 10 & 9 \\
\hline & 400 & 24 & 21 & 10 & 7 \\
\hline & 450 & 22 & 13 & 8 & 6 \\
\hline & 500 & 19 & 15 & 9 & 5 \\
\hline & 550 & 15 & 11 & 7 & 2 \\
\hline & 600 & 16 & 11 & 5 & 2 \\
\hline 30 & 200 & 29 & 23 & 15 & 15 \\
\hline 35 & & 30 & 26 & 17 & 15 \\
\hline 40 & & 32 & 29 & 19 & 16 \\
\hline 45 & & 33 & 32 & 20 & 18 \\
\hline 50 & & 34 & 33 & 25 & 23 \\
\hline 55 & & 36 & 35 & 26 & 24 \\
\hline 60 & & 40 & 36 & 29 & 26 \\
\hline
\end{tabular}


Table 3 (continued)

\begin{tabular}{|c|c|c|c|c|c|}
\hline Nodes & $\begin{array}{l}\text { Transmission } \\
\text { range }\end{array}$ & GA & CLPSO & $\mathrm{ACO}$ & M-SSO \\
\hline \multirow[t]{11}{*}{50} & 100 & 50 & 45 & 35 & 33 \\
\hline & 150 & 50 & 40 & 27 & 29 \\
\hline & 200 & 43 & 44 & 25 & 21 \\
\hline & 250 & 37 & 33 & 22 & 19 \\
\hline & 300 & 35 & 31 & 17 & 14 \\
\hline & 350 & 28 & 26 & 16 & 11 \\
\hline & 400 & 32 & 22 & 13 & 8 \\
\hline & 450 & 28 & 20 & 10 & 6 \\
\hline & 500 & 22 & 18 & 8 & 5 \\
\hline & 550 & 15 & 19 & 7 & 3 \\
\hline & 600 & 16 & 15 & 5 & 3 \\
\hline 30 & 300 & 21 & 19 & 11 & 10 \\
\hline 35 & & 22 & 21 & 12 & 10 \\
\hline 40 & & 23 & 22 & 12 & 11 \\
\hline 45 & & 23 & 22 & 13 & 12 \\
\hline 50 & & 24 & 23 & 14 & 12 \\
\hline 55 & & 26 & 25 & 16 & 13 \\
\hline 60 & & 28 & 26 & 17 & 14 \\
\hline \multirow[t]{9}{*}{60} & 100 & 55 & 50 & 42 & 38 \\
\hline & 150 & 57 & 45 & 33 & 35 \\
\hline & 200 & 50 & 39 & 29 & 26 \\
\hline & 250 & 48 & 33 & 22 & 18 \\
\hline & 300 & 41 & 29 & 19 & 15 \\
\hline & 350 & 35 & 29 & 16 & 13 \\
\hline & 400 & 26 & 25 & 14 & 11 \\
\hline & 450 & 21 & 22 & 10 & 8 \\
\hline & 500 & 20 & 19 & 8 & 5 \\
\hline \multirow[t]{2}{*}{60} & 550 & 21 & 15 & 6 & 5 \\
\hline & 600 & 19 & 12 & 6 & 3 \\
\hline 30 & 400 & 17 & 16 & 8 & 7 \\
\hline 35 & & 17 & 16 & 9 & 8 \\
\hline 40 & & 18 & 17 & 10 & 9 \\
\hline 45 & & 19 & 18 & 9 & 7 \\
\hline 50 & & 20 & 19 & 7 & 6 \\
\hline 55 & & 21 & 18 & 8 & 5 \\
\hline 60 & & 23 & 19 & 9 & 5 \\
\hline
\end{tabular}

\section{Experimentation and performance evaluation}

The proposed algorithm has been implemented in MATLAB version 8.1 with a system configuration of Intel core i7 processor with $3.2 \mathrm{GHz}$ speed and 4 GB RAM in a $500 \mathrm{~GB}$ 
Fig. 10 Clusters vs varied transmission range with fixed nodes 30 of Grid 2X2

Fig. 11 Clusters vs varied transmission range with fixed nodes 40 of Grid 2X2

Fig. 12 Clusters vs varied transmission range with fixed nodes 50 of Grid 2X2

Fig. 13 Clusters vs varied transmission range with fixed nodes 60 of Grid 2X2
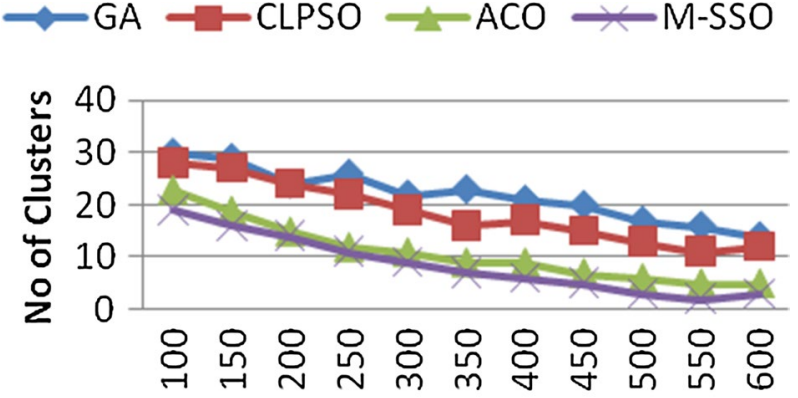

Transmission Range
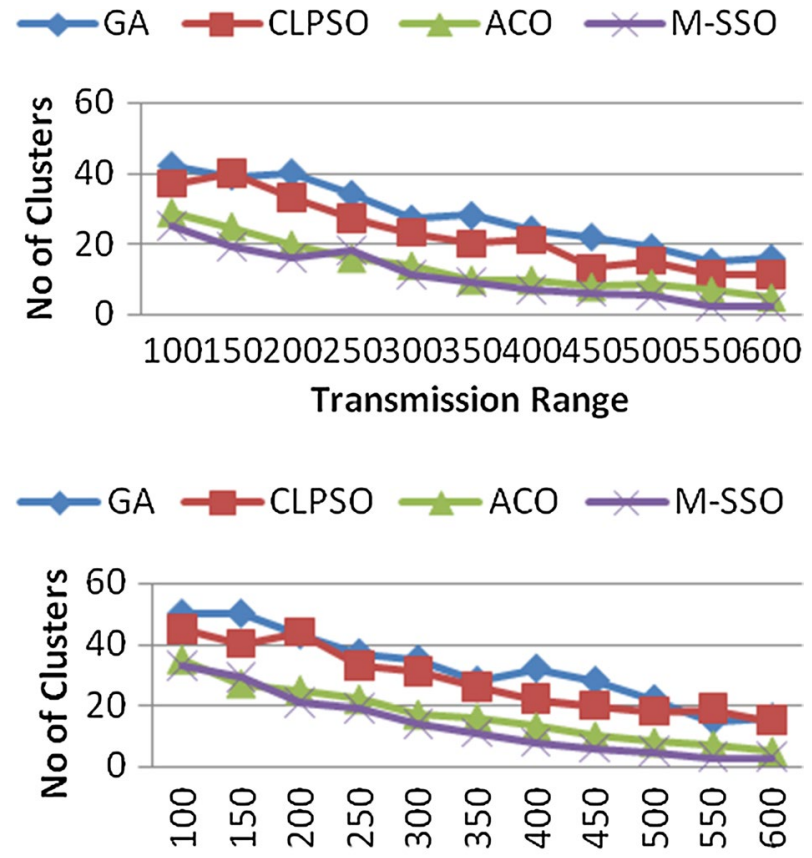

Transmission Range
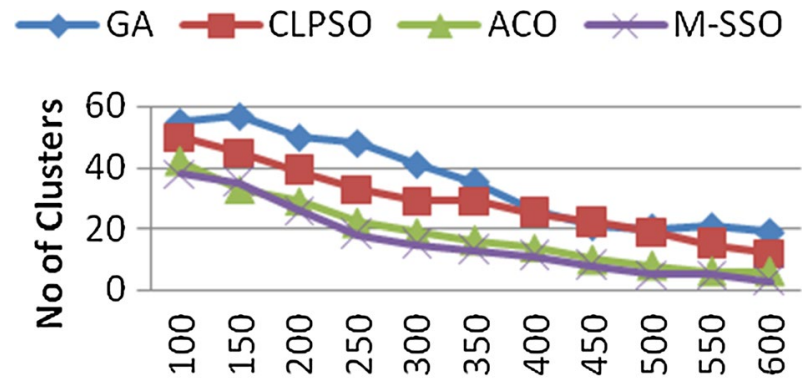

Transmission Range 
Fig. 14 Clusters vs varied \# nodes with fixed transmission range 100 of Grid 2X2

Fig. 15 Clusters vs varied \# nodes with fixed transmission range 200 of Grid $2 X 2$

Fig. 16 Clusters vs varied \# nodes with fixed transmission range 300 of Grid 2X2
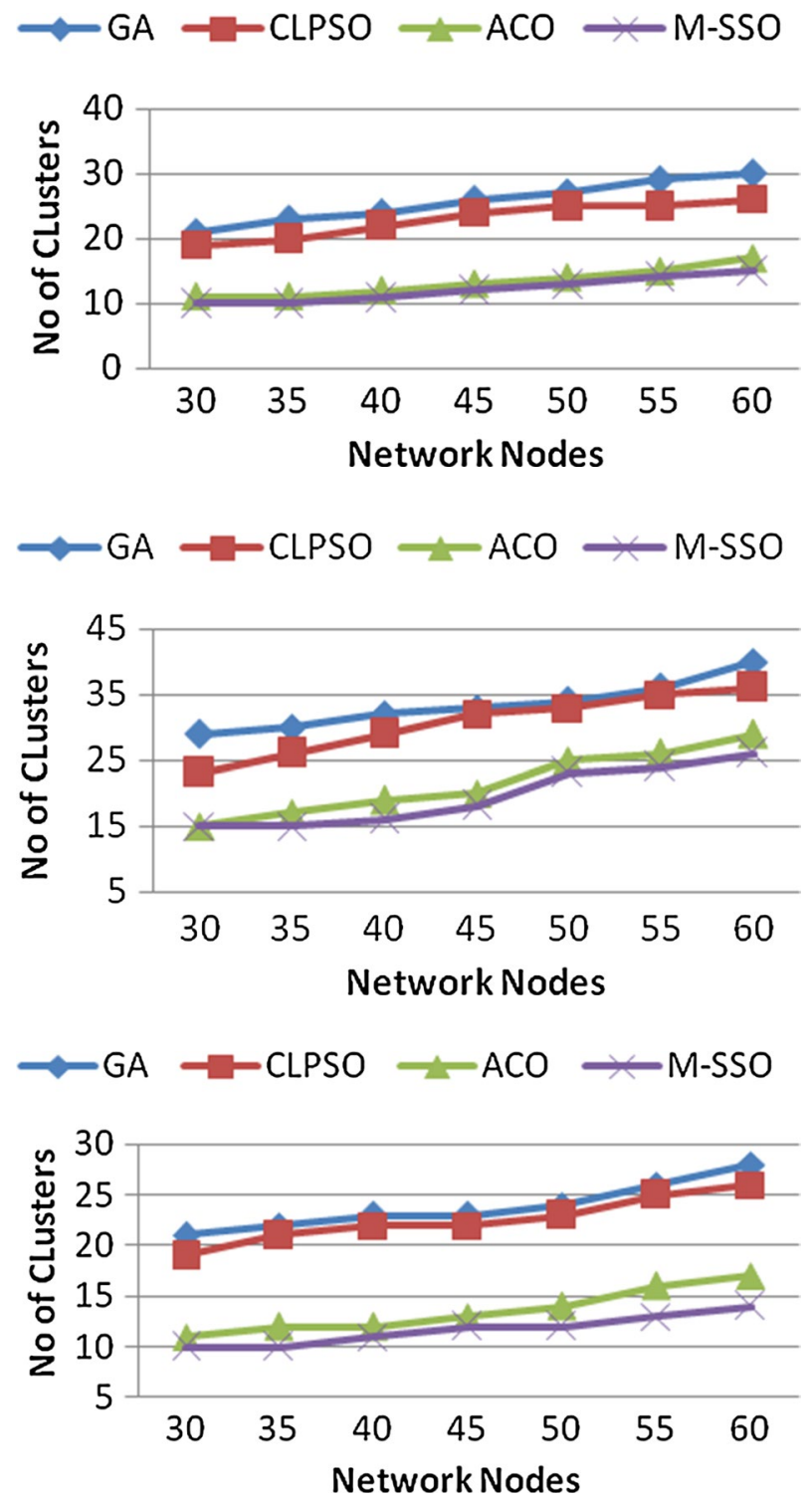

HDD. For comparing the performance of the proposed algorithm, a simulation has been conducted on the existing algorithms in the same environmental setup. The parameters are tabulated in Table 1. This table includes the parameters of the simulation region and also the proposed algorithm.

Observation 5 The test bed has been designed in MATLAB with a road of 2 lanes running in opposite directions. The vehicles that travel in one lane are assumed to not change their lane. The transmission range is for vehicles with a varied range of 100 to 600 . The initial velocity is given as the experimental analyses have been carried out in two scenarios: 
Fig. 17 Clusters vs varied \# nodes with fixed transmission range 400 of Grid $2 X 2$

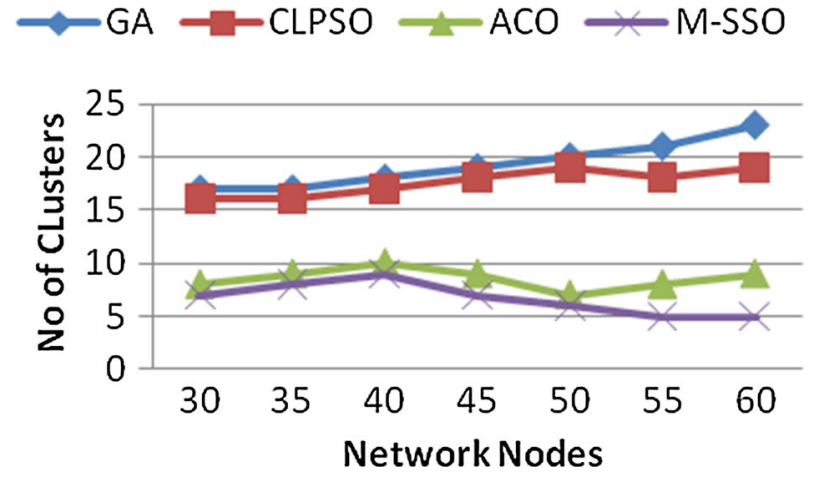

varied transmission range vs. number of clusters and varied number of nodes vs. number of clusters. Each scenario has been tested in four different grids, each with a different and increased size for the simulation region. Data sets used to test the proposed approach, i-VANET, cooperative localization in vehicular networks (CLVN), distributed denial of service data sets and taxi trace datasets are taken into consideration for best suites the IoT, sensors, signal processing, GPS receiver, vehicle communication limitation to approximately 10 to 12 Kilometers.

Observation 6 Table 2 presents the results obtained from the simulation of the proposed and existing algorithms with a varied transmission range but with a fixed number of nodes and also with a varied number of nodes but with a fixed transmission range for grid 1X1. The comparisons between each algorithm are given from Figs. 2, 3, 4, 5, 6, 7, 8 and 9.

Observation 7 Figures 2, 3, 4 and 5 compare the result obtained for the formation of the clusters with a varied transmission range but with a fixed number of nodes for the grid 1X1. The results show that as the transmission range increases the proposed algorithm outperforms the existing algorithms. For the transmission range of 550-600, the existing algorithm ACO and the proposed M-SSO perform equally well in clustering the vehicles. Figures 6, 7, 8 and 9 compare the results obtained for the formation of the clusters with a varied number of nodes and a fixed transmission range for the grid $1 \mathrm{X} 1$. The results show that as the number of nodes increases the proposed algorithm outperforms the existing algorithms. When the number of nodes is 5.5, ACO and the proposed M-SSO perform equally well in clustering the vehicles.

Observation 8 Table 3 presents the results obtained from the simulation of the proposed and existing algorithms with a varied transmission range but with a fixed number of nodes and also with a varied number of nodes but with a fixed transmission range for grid $2 \mathrm{X} 2$. The comparisons between each algorithm are given from Figs.10, 11, 12, 13, 14, 15, 16 and 17.

Observation 9 Figures 10,11, 12 and 13 compare the result obtained for the formation of the clusters with a varied transmission range but with a fixed number of nodes for the grid $2 \mathrm{X} 2$. The results show that as the transmission range increases the proposed algorithm outperforms the existing algorithms by obtaining the minimum number of clusters. Over a wider transmission range both ACO and M-SSO outperform other existing algorithms. 
Table 4 Results of total number of clusters of different algorithms for grid $3 \mathrm{X} 3$

\begin{tabular}{|c|c|c|c|c|c|}
\hline Nodes & $\begin{array}{l}\text { Transmission } \\
\text { range }\end{array}$ & GA & CLPSO & $\mathrm{ACO}$ & M-SSO \\
\hline \multirow[t]{11}{*}{30} & 100 & 35 & 30 & 24 & 20 \\
\hline & 150 & 33 & 32 & 22 & 19 \\
\hline & 200 & 32 & 28 & 17 & 18 \\
\hline & 250 & 29 & 24 & 15 & 13 \\
\hline & 300 & 27 & 20 & 15 & 11 \\
\hline & 350 & 25 & 19 & 14 & 9 \\
\hline & 400 & 20 & 17 & 11 & 9 \\
\hline & 450 & 22 & 16 & 11 & 7 \\
\hline & 500 & 20 & 18 & 10 & 5 \\
\hline & 550 & 19 & 15 & 9 & 7 \\
\hline & 600 & 18 & 15 & 9 & 5 \\
\hline 30 & 100 & 30 & 30 & 24 & 22 \\
\hline 35 & & 35 & 35 & 28 & 25 \\
\hline 40 & & 40 & 37 & 32 & 28 \\
\hline 45 & & 47 & 43 & 35 & 31 \\
\hline 50 & & 50 & 48 & 37 & 35 \\
\hline 55 & & 56 & 55 & 41 & 36 \\
\hline 60 & & 60 & 58 & 47 & 39 \\
\hline \multirow[t]{11}{*}{40} & 100 & 40 & 38 & 33 & 30 \\
\hline & 150 & 39 & 36 & 29 & 22 \\
\hline & 200 & 38 & 33 & 27 & 25 \\
\hline & 250 & 34 & 30 & 23 & 19 \\
\hline & 300 & 32 & 28 & 20 & 17 \\
\hline & 350 & 30 & 25 & 19 & 15 \\
\hline & 400 & 28 & 22 & 17 & 16 \\
\hline & 450 & 26 & 23 & 13 & 7 \\
\hline & 500 & 25 & 21 & 11 & 8 \\
\hline & 550 & 22 & 19 & 10 & 6 \\
\hline & 600 & 20 & 17 & 10 & 6 \\
\hline 30 & 200 & 30 & 29 & 17 & 15 \\
\hline 35 & & 34 & 31 & 22 & 18 \\
\hline 40 & & 36 & 33 & 27 & 23 \\
\hline 45 & & 38 & 37 & 28 & 26 \\
\hline 50 & & 45 & 42 & 31 & 28 \\
\hline 55 & & 49 & 46 & 35 & 31 \\
\hline 60 & & 52 & 47 & 37 & 35 \\
\hline
\end{tabular}


A. Shankar et al.

Table 4 (continued)

\begin{tabular}{|c|c|c|c|c|c|}
\hline Nodes & $\begin{array}{l}\text { Transmission } \\
\text { range }\end{array}$ & GA & CLPSO & $\mathrm{ACO}$ & M-SSO \\
\hline \multirow[t]{11}{*}{50} & 100 & 50 & 48 & 39 & 34 \\
\hline & 150 & 45 & 42 & 35 & 29 \\
\hline & 200 & 46 & 39 & 33 & 31 \\
\hline & 250 & 42 & 35 & 29 & 25 \\
\hline & 300 & 39 & 30 & 24 & 20 \\
\hline & 350 & 31 & 25 & 22 & 17 \\
\hline & 400 & 27 & 21 & 17 & 11 \\
\hline & 450 & 23 & 18 & 14 & 9 \\
\hline & 500 & 24 & 14 & 11 & 7 \\
\hline & 550 & 22 & 13 & 9 & 5 \\
\hline & 600 & 22 & 14 & 9 & 5 \\
\hline 30 & 300 & 29 & 24 & 17 & 15 \\
\hline 35 & & 28 & 26 & 20 & 17 \\
\hline 40 & & 27 & 30 & 22 & 20 \\
\hline 45 & & 31 & 32 & 25 & 22 \\
\hline 50 & & 37 & 34 & 27 & 24 \\
\hline 55 & & 40 & 39 & 28 & 28 \\
\hline 60 & & 45 & 42 & 30 & 29 \\
\hline \multirow[t]{9}{*}{60} & 100 & 62 & 59 & 49 & 40 \\
\hline & 150 & 59 & 55 & 42 & 43 \\
\hline & 200 & 61 & 49 & 38 & 35 \\
\hline & 250 & 58 & 35 & 29 & 26 \\
\hline & 300 & 49 & 33 & 30 & 22 \\
\hline & 350 & 35 & 29 & 27 & 23 \\
\hline & 400 & 28 & 24 & 22 & 18 \\
\hline & 450 & 25 & 22 & 19 & 12 \\
\hline & 500 & 24 & 21 & 15 & 11 \\
\hline \multirow[t]{2}{*}{60} & 550 & 22 & 20 & 11 & 7 \\
\hline & 600 & 22 & 20 & 9 & 5 \\
\hline 30 & 400 & 23 & 21 & 14 & 11 \\
\hline 35 & & 23 & 23 & 15 & 12 \\
\hline 40 & & 24 & 24 & 17 & 13 \\
\hline 45 & & 27 & 26 & 16 & 14 \\
\hline 50 & & 30 & 28 & 17 & 16 \\
\hline 55 & & 31 & 30 & 19 & 17 \\
\hline 60 & & 33 & 31 & 20 & 18 \\
\hline
\end{tabular}

However, over a narrower transmission range our proposed algorithm outperforms all other existing algorithms. 
Fig. 18 Clusters vs varied transmission range with fixed nodes 30 of Grid $3 \times 3$

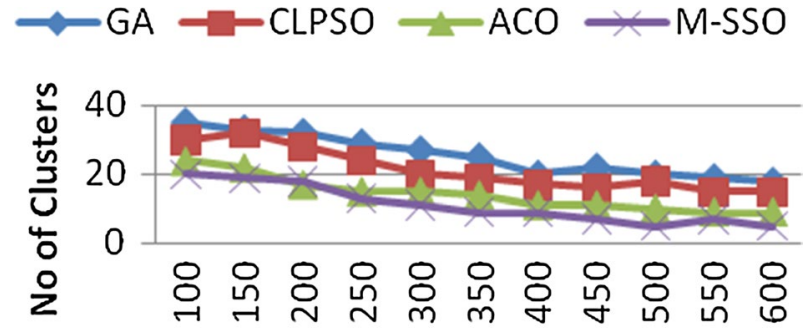

Transmission Range

Fig. 19 Clusters vs varied transmission range with fixed nodes 40 of Grid $3 \times 3$

Fig. 20 Clusters vs varied transmission range with fixed nodes 50 of Grid $3 \times 3$

Fig. 21 Clusters vs Varied Transmission Range with Fixed nodes 60 of Grid 3X3
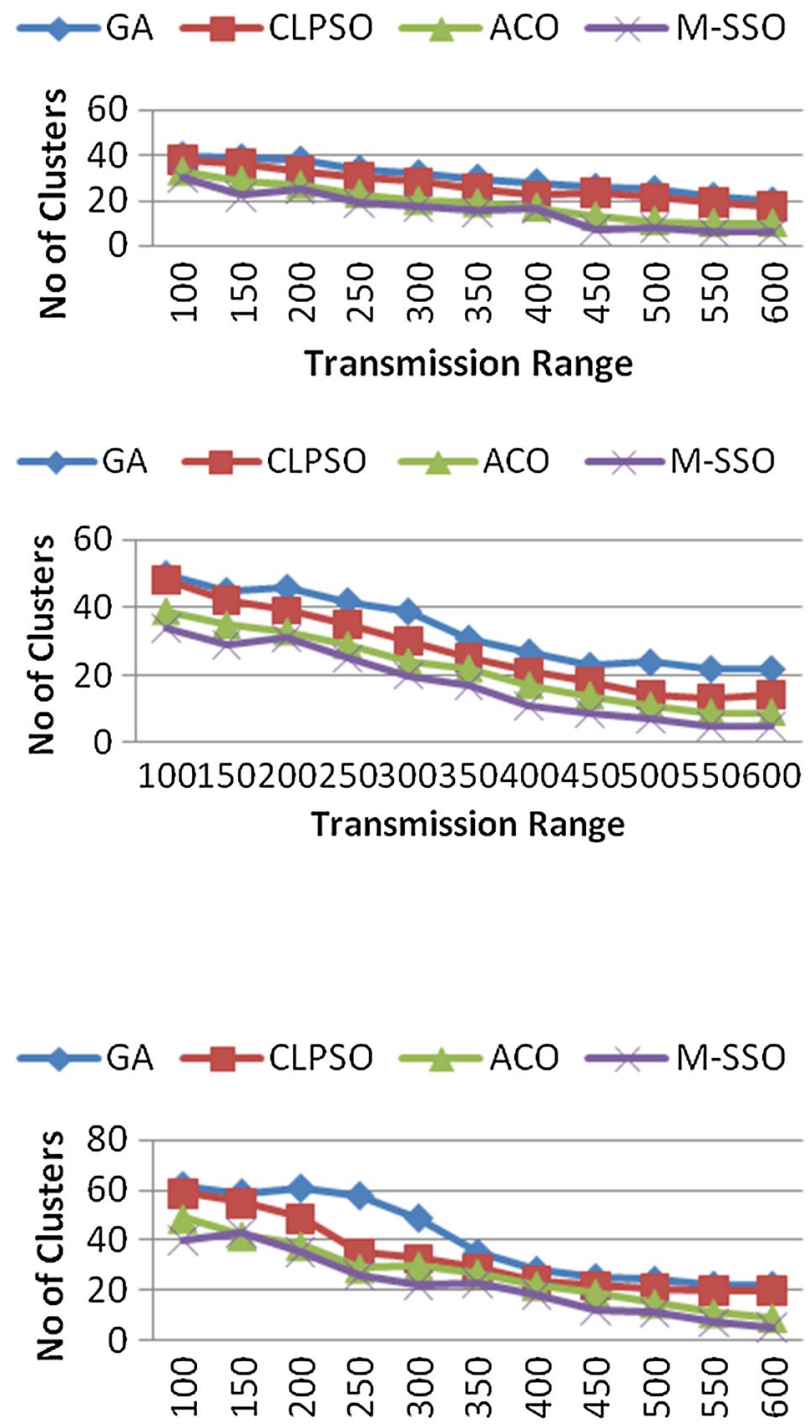

Transmission Range 
Fig. 22 Clusters vs varied \# nodes with fixed transmission range 100 of Grid 3X3

Fig. 23 Clusters vs varied \# nodes with fixed transmission range 200 of Grid $3 X 3$

Fig. 24 Clusters vs varied \# nodes with fixed transmission range 300 of Grid $3 \times 3$

Fig. 25 Clusters vs varied \# nodes with fixed transmission range 400 of Grid $3 \times 3$
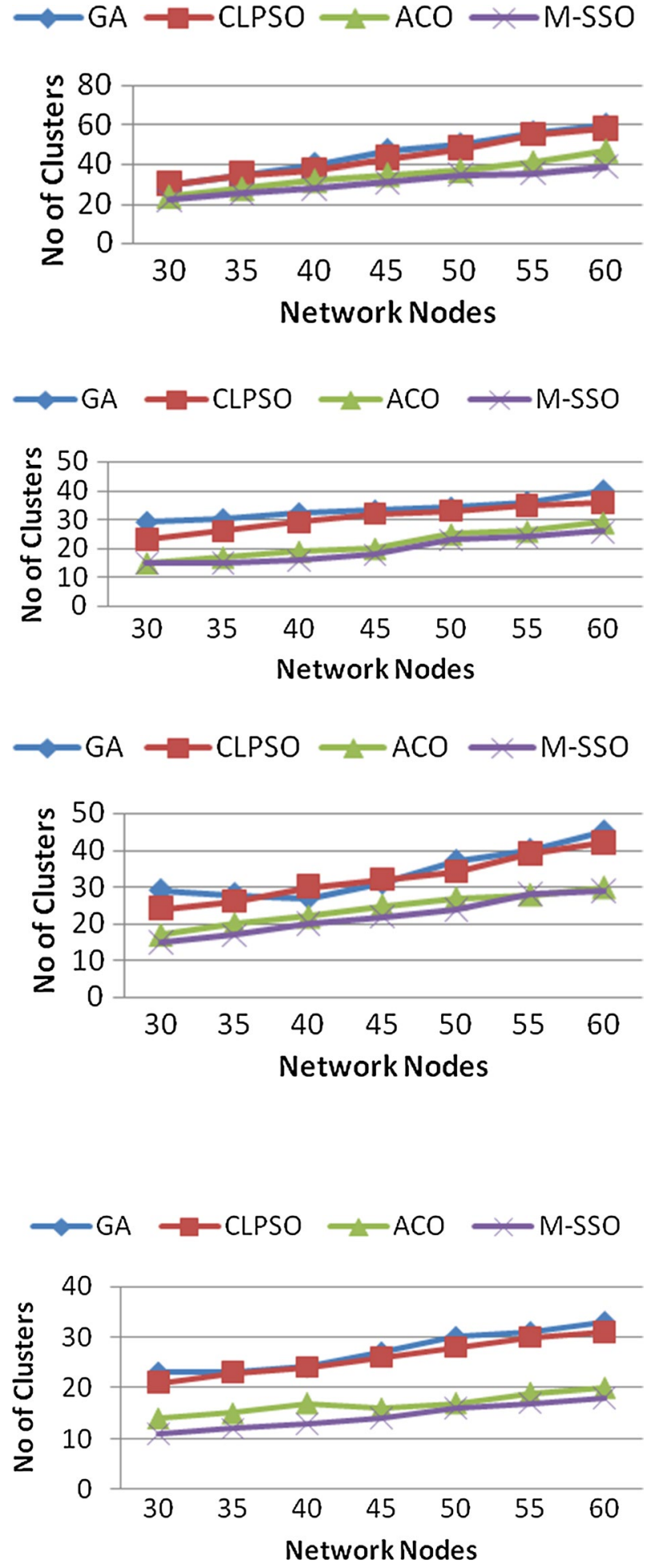
Table 5 Results of total number of clusters of different algorithms for grid 4X4

\begin{tabular}{|c|c|c|c|c|c|}
\hline Nodes & $\begin{array}{l}\text { Transmission } \\
\text { range }\end{array}$ & GA & CLPSO & $\mathrm{ACO}$ & M-SSO \\
\hline \multirow[t]{11}{*}{30} & 100 & 35 & 30 & 24 & 20 \\
\hline & 150 & 35 & 29 & 23 & 18 \\
\hline & 200 & 32 & 25 & 20 & 18 \\
\hline & 250 & 29 & 24 & 24 & 19 \\
\hline & 300 & 27 & 21 & 18 & 15 \\
\hline & 350 & 28 & 20 & 16 & 14 \\
\hline & 400 & 24 & 18 & 17 & 15 \\
\hline & 450 & 27 & 19 & 15 & 13 \\
\hline & 500 & 24 & 17 & 14 & 10 \\
\hline & 550 & 23 & 16 & 15 & 11 \\
\hline & 600 & 22 & 16 & 13 & 10 \\
\hline 30 & 100 & 30 & 30 & 26 & 22 \\
\hline 35 & & 39 & 37 & 28 & 26 \\
\hline 40 & & 42 & 40 & 37 & 32 \\
\hline 45 & & 48 & 44 & 40 & 35 \\
\hline 50 & & 53 & 50 & 42 & 40 \\
\hline 55 & 100 & 57 & 56 & 46 & 43 \\
\hline 60 & & 60 & 59 & 49 & 45 \\
\hline \multirow[t]{8}{*}{40} & 100 & 40 & 40 & 34 & 33 \\
\hline & 150 & 40 & 38 & 31 & 31 \\
\hline & 200 & 39 & 36 & 32 & 29 \\
\hline & 250 & 35 & 32 & 29 & 26 \\
\hline & 300 & 36 & 30 & 25 & 22 \\
\hline & 350 & 33 & 31 & 23 & 19 \\
\hline & 400 & 30 & 28 & 21 & 16 \\
\hline & 450 & 28 & 25 & 17 & 12 \\
\hline \multirow[t]{3}{*}{40} & 500 & 25 & 26 & 16 & 11 \\
\hline & 550 & 23 & 24 & 15 & 10 \\
\hline & 600 & 24 & 23 & 14 & 10 \\
\hline 30 & 200 & 33 & 29 & 22 & 20 \\
\hline 35 & & 38 & 33 & 26 & 25 \\
\hline 40 & & 40 & 39 & 31 & 28 \\
\hline 45 & & 46 & 42 & 35 & 31 \\
\hline 50 & & 48 & 45 & 37 & 33 \\
\hline 55 & & 55 & 49 & 40 & 37 \\
\hline 60 & & 58 & 54 & 42 & 40 \\
\hline
\end{tabular}


A. Shankar et al.

Table 5 (continued)

\begin{tabular}{|c|c|c|c|c|c|}
\hline Nodes & $\begin{array}{l}\text { Transmission } \\
\text { range }\end{array}$ & GA & CLPSO & $\mathrm{ACO}$ & M-SSO \\
\hline \multirow[t]{11}{*}{50} & 100 & 50 & 50 & 45 & 38 \\
\hline & 150 & 49 & 47 & 40 & 39 \\
\hline & 200 & 46 & 43 & 36 & 33 \\
\hline & 250 & 44 & 39 & 34 & 30 \\
\hline & 300 & 42 & 35 & 29 & 26 \\
\hline & 350 & 38 & 31 & 27 & 22 \\
\hline & 400 & 37 & 29 & 24 & 19 \\
\hline & 450 & 32 & 26 & 21 & 15 \\
\hline & 500 & 31 & 25 & 17 & 19 \\
\hline & 550 & 29 & 26 & 15 & 12 \\
\hline & 600 & 29 & 24 & 14 & 10 \\
\hline 30 & 300 & 30 & 27 & 22 & 20 \\
\hline 35 & & 32 & 30 & 25 & 24 \\
\hline 40 & & 37 & 34 & 27 & 25 \\
\hline 45 & & 38 & 36 & 28 & 25 \\
\hline 50 & & 40 & 38 & 29 & 26 \\
\hline 55 & & 49 & 43 & 33 & 30 \\
\hline 60 & & 55 & 45 & 38 & 35 \\
\hline \multirow[t]{7}{*}{60} & 100 & 60 & 56 & 49 & 45 \\
\hline & 150 & 57 & 50 & 46 & 46 \\
\hline & 200 & 58 & 51 & 43 & 39 \\
\hline & 250 & 50 & 45 & 39 & 32 \\
\hline & 300 & 49 & 43 & 35 & 29 \\
\hline & 350 & 45 & 39 & 32 & 26 \\
\hline & 400 & 41 & 32 & 29 & 22 \\
\hline \multirow[t]{4}{*}{60} & 450 & 38 & 30 & 25 & 16 \\
\hline & 500 & 35 & 29 & 20 & 13 \\
\hline & 550 & 32 & 29 & 17 & 10 \\
\hline & 600 & 31 & 28 & 15 & 10 \\
\hline 30 & 400 & 25 & 23 & 20 & 16 \\
\hline 35 & & 29 & 26 & 22 & 19 \\
\hline 40 & & 32 & 29 & 25 & 22 \\
\hline 45 & 400 & 31 & 32 & 27 & 23 \\
\hline 50 & & 30 & 34 & 28 & 24 \\
\hline 55 & & 36 & 35 & 29 & 25 \\
\hline 60 & & 40 & 37 & 31 & 27 \\
\hline
\end{tabular}

Observation 10 Figures 14, 15, 16 and 17 compare the result obtained for the formation of clusters with a varied number of nodes but with a fixed transmission range for the grid $2 \mathrm{X} 2$. The results show that as the number of nodes increases the proposed algorithm outperforms the existing algorithms. The proposed algorithm obtains similar results to those 
Fig. 26 Clusters vs varied transmission range with fixed nodes 30 of Grid 4 X4

Fig. 27 Clusters vs varied transmission range with fixed nodes 40 of Grid 4 X4

Fig. 28 Clusters vs varied transmission range with fixed nodes 50 of Grid 4 X4

Fig. 29 Clusters vs varied transmission range with fixed nodes 60 of Grid $4 \times 4$
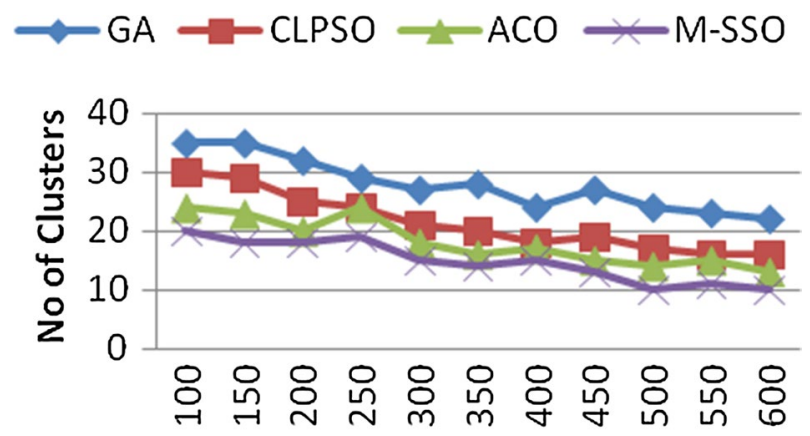

Transmission Range

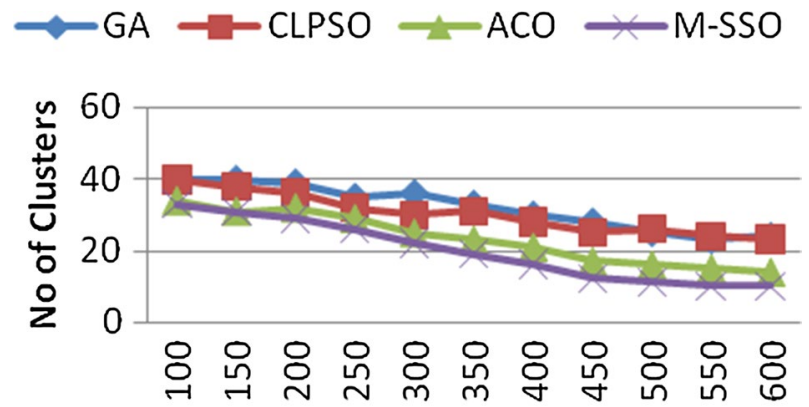

Transmission Range
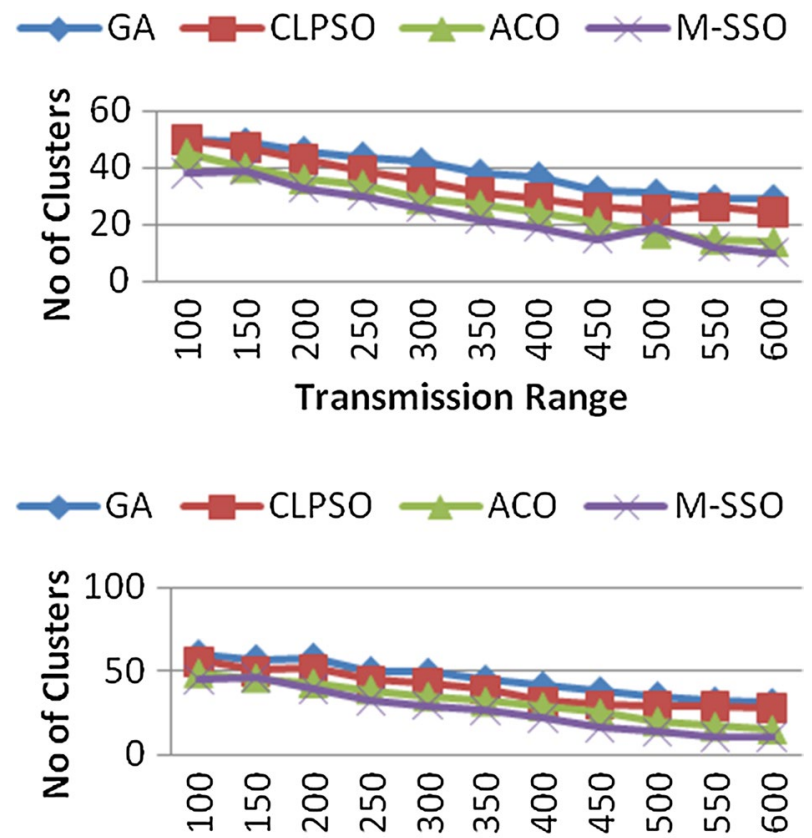

Transmission Range 
Fig. 30 Clusters vs varied \# nodes with fixed transmission range 100 of Grid 4X4

Fig. 31 Clusters vs varied \# nodes with fixed transmission range 200 of Grid 4X4

Fig. 32 Clusters vs varied \# nodes with fixed transmission range 300 of Grid 4X4

Fig. 33 Clusters vs varied \# nodes with fixed transmission range 400 of Grid 4X4
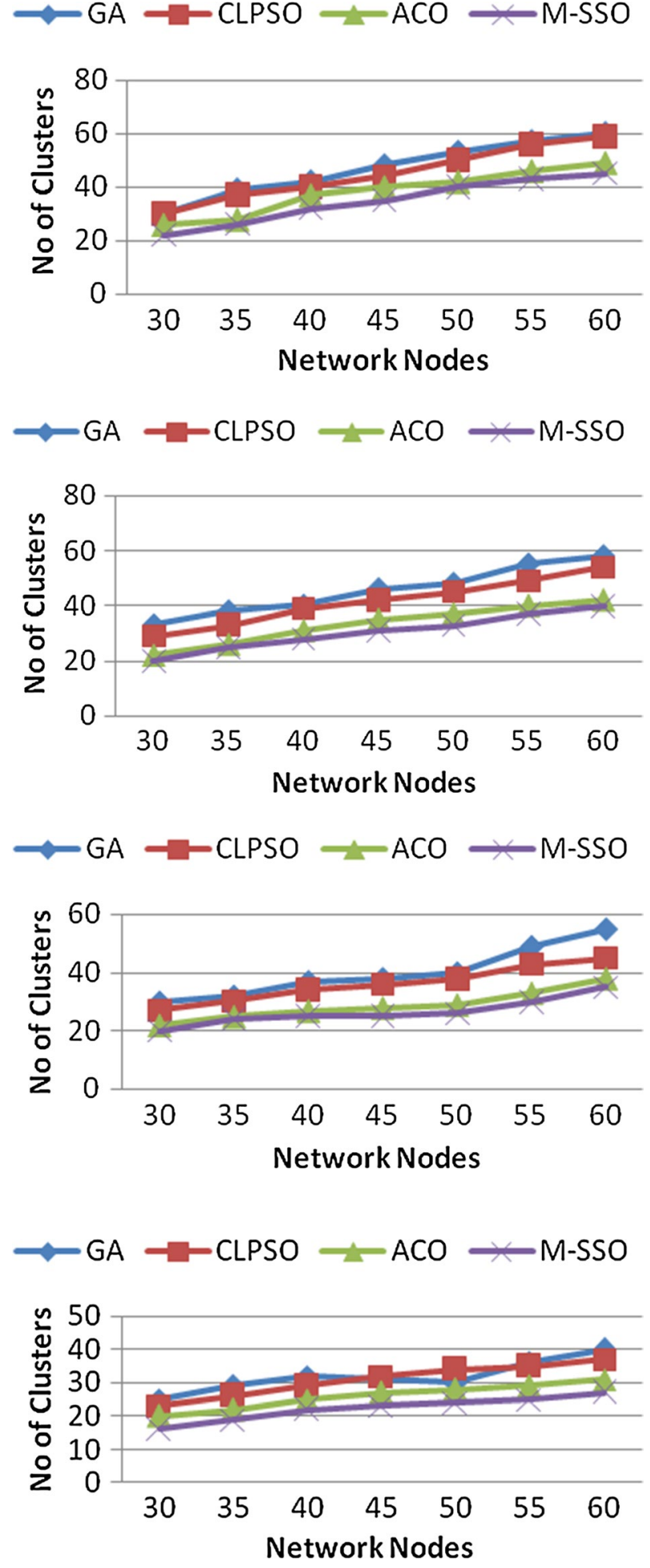
of ACO in the case of fewer network nodes but as the size of the network nodes increases (Ed-either 'as the size of the network increases' or 'as the number of nodes increases') it outperforms existing algorithms.

Observation 11 Table 4 presents the results obtained from the simulation of the proposed and existing algorithms with a varied transmission range but with a fixed number of nodes and also with a varied number of nodes but with a fixed transmission range for grid $3 \mathrm{X} 3$. Each algorithm is compared in Figs.18, 19, 20, 21, 22, 23, 24 and 25.

Observation 12 Figures 18, 19, 20 and 21 compare the result obtained for the formation of the clusters with a varied transmission range but with a fixed number of nodes for the grid 3X3. On calculating the correlation factor between each set of results with respect to all the algorithms, our proposed M-SSO outperforms all other existing algorithms.

Fig.34 Comparison of throughput for varied packet size of grid $1 \times 1$

Fig. 35 Comparison of throughput for varied packet size of grid 2X2

Fig. 36 Comparison of throughput for varied packet size of grid 3X3
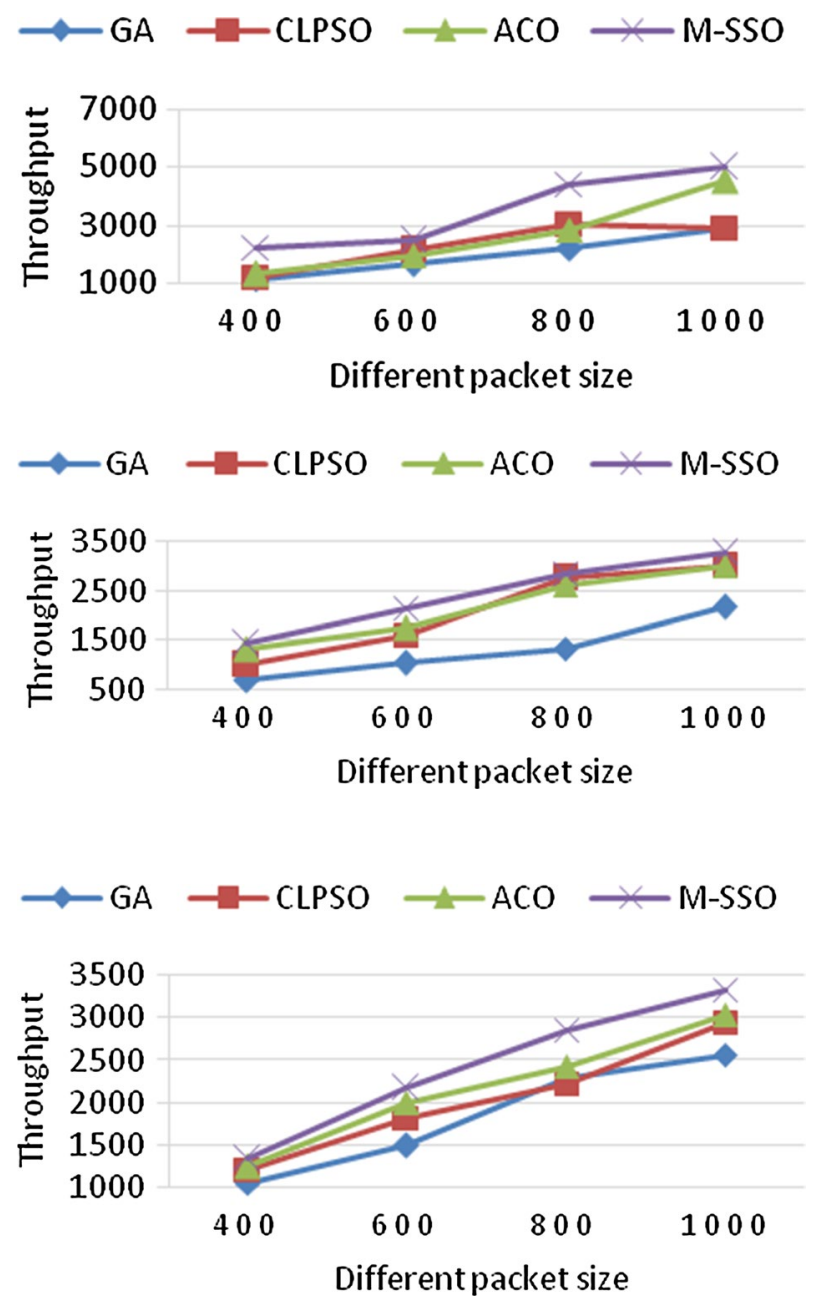
Fig. 37 Comparison of throughput for varied packet size of grid $4 \mathrm{X} 4$

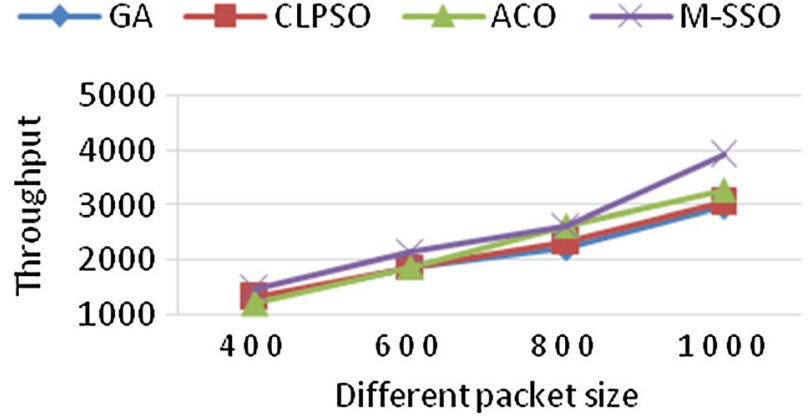

Table 6 Simulation results of algorithms under different regions with varied grid size, packet size and number of nodes w.r.t. throughput

\begin{tabular}{|c|c|c|c|c|c|c|}
\hline Grid size (Km.) & $\begin{array}{l}\text { Packet size } \\
\text { (Bytes) }\end{array}$ & No. of nodes & GA & CLPSO & $\mathrm{ACO}$ & M-SSO \\
\hline \multirow[t]{8}{*}{$1 \mathrm{X} 1$} & 400 & 50 & 1176.47 & 1250.00 & 1356.33 & 2264.22 \\
\hline & 600 & & 1666.67 & 2142.86 & 2000.00 & 2500.00 \\
\hline & 800 & & 2222.22 & 3076.92 & 2857.14 & 4438.44 \\
\hline & 1000 & & 2941.18 & 2941.18 & 4545.45 & 5000.00 \\
\hline & 512 & 30 & 808.42 & 903.53 & 1296.36 & 1280.00 \\
\hline & & 40 & 1280.00 & 1204.71 & 1462.86 & 1575.38 \\
\hline & & 50 & 1193.00 & 1280.00 & 1505.88 & 1969.23 \\
\hline & & 60 & 1536.00 & 1706.67 & 1920.00 & 2048.00 \\
\hline \multirow[t]{8}{*}{$2 \times 2$} & 400 & 50 & 689.66 & 1000.00 & 1304.35 & 1428.57 \\
\hline & 600 & & 1034.48 & 1607.14 & 1730.77 & 2142.86 \\
\hline & 800 & & 1333.33 & 2742.86 & 2608.70 & 2857.14 \\
\hline & 1000 & & 2162.50 & 2977.78 & 3000.00 & 3260.87 \\
\hline & 512 & 30 & 529.66 & 512.00 & 614.40 & 698.18 \\
\hline & & 40 & 731.43 & 682.67 & 819.20 & 975.24 \\
\hline & & 50 & 853.33 & 984.62 & 984.62 & 1219.05 \\
\hline & & 60 & 1137.78 & 1059.31 & 1280.00 & 1335.65 \\
\hline \multirow[t]{8}{*}{$3 \times 3$} & 400 & 50 & 1052.63 & 1212.12 & 1250.00 & 1333.33 \\
\hline & 600 & & 1500.00 & 1818.18 & 2000.00 & 2164.71 \\
\hline & 800 & & 2285.71 & 2222.22 & 2424.24 & 2857.14 \\
\hline & 1000 & & 2564.10 & 2941.18 & 3030.30 & 3330.30 \\
\hline & 512 & 30 & 384.00 & 465.45 & 495.48 & 451.76 \\
\hline & & 40 & 512.00 & 620.61 & 620.61 & 640.00 \\
\hline & & 50 & 656.41 & 731.43 & 853.33 & 752.94 \\
\hline & & 60 & 808.42 & 808.42 & 1024.00 & 1024.00 \\
\hline \multirow[t]{8}{*}{$4 X 4$} & 400 & 50 & 1250.00 & 1315.79 & 1219.51 & 1470.59 \\
\hline & 600 & & 1875.00 & 1875.00 & 1875.00 & 2173.68 \\
\hline & 800 & & 2222.22 & 2325.58 & 2631.58 & 2631.58 \\
\hline & 1000 & & 2976.19 & 3048.78 & 3289.47 & 3906.25 \\
\hline & 512 & 30 & 374.63 & 365.71 & 404.21 & 404.21 \\
\hline & & 40 & 476.28 & 476.28 & 525.13 & 568.89 \\
\hline & & 50 & 568.89 & 595.35 & 673.68 & 691.89 \\
\hline & & 60 & 749.27 & 698.18 & 787.69 & 990.97 \\
\hline
\end{tabular}


Observation 13 Figures 22, 23, 24 and 25 compare the result obtained for the formation of clusters with a varied number of nodes but with a fixed transmission range for the grid $3 \mathrm{X} 3$. The results show that as the number of nodes increases the proposed algorithm outperforms the existing algorithms. The proposed algorithm obtains similar results to those of ACO under the condition of fewer network nodes but as the size of the number of nodes increases it outperforms existing algorithms.

Observation 14 Table 5 presents the results obtained from the simulation of the proposed and existing algorithms with a varied transmission range but with a fixed number of nodes and also with a varied number of nodes but with a fixed transmission range for grid $4 \mathrm{X} 4$. Each algorithm is compared in Figs. 26, 27, 28, 29, 30, 31, 32 and 33.

Table7 Efficiency evaluation and its comparison with relevant approaches

\begin{tabular}{|c|c|c|c|c|c|c|}
\hline Grid Size (Km.) & $\begin{array}{l}\text { Packet Size } \\
\text { (Bytes) }\end{array}$ & No. of Nodes & CLPSO & $\mathrm{ACO}$ & M-SSO & Auto-Op \\
\hline \multirow[t]{8}{*}{$1 \mathrm{X} 1$} & 400 & 50 & 19 & 18 & 16 & 13 \\
\hline & 600 & & 22 & 20 & 18 & 16 \\
\hline & 800 & & 26 & 24 & 20 & 15 \\
\hline & 1000 & & 32 & 30 & 26 & 18 \\
\hline & 512 & 30 & 18 & 14 & 13 & 10 \\
\hline & & 40 & 18 & 14 & 13 & 11 \\
\hline & & 50 & 20 & 18 & 15 & 15 \\
\hline & & 60 & 21 & 22 & 19 & 16 \\
\hline \multirow[t]{8}{*}{$2 \mathrm{X} 2$} & 400 & 50 & 27 & 25 & 25 & 21 \\
\hline & 600 & & 31 & 29 & 28 & 22 \\
\hline & 800 & & 33 & 30 & 31 & 28 \\
\hline & 1000 & & 36 & 34 & 31 & 32 \\
\hline & 512 & 30 & 26 & 22 & 22 & 20 \\
\hline & & 40 & 28 & 26 & 26 & 23 \\
\hline & & 50 & 34 & 32 & 29 & 25 \\
\hline & & 60 & 37 & 34 & 29 & 27 \\
\hline \multirow[t]{8}{*}{$3 \times 3$} & 400 & 50 & 37 & 33 & 29 & 25 \\
\hline & 600 & & 45 & 38 & 29 & 27 \\
\hline & 800 & & 52 & 55 & 37 & 36 \\
\hline & 1000 & & 62 & 52 & 48 & 42 \\
\hline & 512 & 30 & 36 & 31 & 30 & 28 \\
\hline & & 40 & 44 & 40 & 29 & 25 \\
\hline & & 50 & 57 & 47 & 39 & 37 \\
\hline & & 60 & 60 & 52 & 45 & 42 \\
\hline \multirow[t]{8}{*}{$4 X 4$} & 400 & 50 & 44 & 39 & 28 & 25 \\
\hline & 600 & & 54 & 45 & 33 & 29 \\
\hline & 800 & & 61 & 49 & 41 & 39 \\
\hline & 1000 & & 69 & 56 & 49 & 45 \\
\hline & 512 & 30 & 41 & 39 & 30 & 27 \\
\hline & & 40 & 58 & 46 & 38 & 31 \\
\hline & & 50 & 55 & 54 & 40 & 39 \\
\hline & & 60 & 68 & 57 & 52 & 49 \\
\hline
\end{tabular}


Observation 15 Figures 26, 27, 28 and 29 compare the result obtained for the formation of the clusters with a varied transmission range but with a fixed number of nodes for the grid $4 \mathrm{X} 4$. The results show that the total number of clusters reduced as the transmission range increases and that the proposed algorithm outperforms existing algorithms.

Observation 16 Figures 30, 31, 32 and 33 compare the result obtained for the formation of the clusters with a varied number of nodes but with a fixed transmission range for the grid $4 \mathrm{X} 4$. The figures show that although the number of clusters increases as the number of nodes increases, the performance of the proposed algorithm is consistent in comparison with the results of existing algorithms. The observation is observed from the first scenario of the proposed work, which is the M-SSO for efficient cluster head selection in VANET. The objective of this chapter is to find the best cluster head selection. The optimal result is selected based on the complete coverage of all vehicles in the VANET scenario as a secondary factor. The proposed methodology has been clearly defined and the quality assessed in terms of the number of clusters under different test bed scenarios. The proposed algorithm has been compared with ACO, PSO and GA, and the results show that the proposed algorithm outperforms the other existing algorithms.

The results of the proposed algorithm are tabulated in terms of packet delivery ratio (PDR), throughput, communication overhead and end-to-end delay. PDR is defined as the ratio between the total number of packets sent to be received by the end user to the number of packets actually received by the end user and this can be formulated as $\operatorname{PDR}=\frac{\sum_{\mathrm{i}=1}^{\mathrm{N}} \text { PacketReceived }_{\mathrm{i}}}{\sum_{\mathrm{i}=1}^{\mathrm{N}} \text { Packetsent }_{\mathrm{i}}}$

Throughput is defined as the total number of packets or bytes transmitted successfully per unit time. This can be formulated as Throughput $=\frac{\sum_{i=1}^{2} \text { PacketTransmitted }_{i}}{\text { unittime }}$.

Routing overhead is defined as the ratio between the total numbers of packet data sent to the destination to the data received by the end user. It has been formulated as Overhead $=\frac{\sum_{\mathrm{i}=1}^{\mathrm{N}} \text { RoutingPacket }_{\mathrm{i}}}{\sum_{\mathrm{i}=1}^{\mathrm{N}} \text { Datapacketreceived }_{\mathrm{i}}}$

End-to-end delay is defined as the total number of times taken by all the packets to reach the defined destination. Delay $=\frac{1}{\mathrm{~N}} \sum_{\mathrm{i}=1}^{\mathrm{N}}\left(\mathrm{T}_{\text {PacketReceived }}^{\mathrm{i}}-\mathrm{T}_{\text {Packetsent }}^{\mathrm{i}}\right)$

As discussed in the previous sections, the experimental analyses for this scenario of the proposed work have been carried using the B-SSO for data discrimination in VANET. Each scenario has been evaluated w.r.t. four different performance metrics as aforementioned. The proposed algorithm has been analyzed for all four grids of the simulation region with a varied number of nodes and with a varied number of packet sizes. The results are tabulated in Table 0.6 and compared in Figs. 34, 35, 36 and 37.

Observation 17 Table 6 represents the results obtained by simulating the proposed algorithm M-SSO, along with three other existing algorithms, GA, CLPSO and ACO, for different simulation regions with a varied number of vehicles and for different packet sizes. For the varied packet size the fixed number of nodes is 50 and for the varied simulation of each set of vehicles the packet size is fixed at 512 bytes.

Observation 18 Figures 34, 35, 36 and 37 compare the results tabulated in Table 6 with respect to different packet sizes on each grid in terms of throughput. The proposed algorithm outperforms the existing algorithms, especially with large packet size. Although all algorithms perform similarly with only marginal difference with small packet size, as the packet size increases the throughput results degrade in all the 4 different sized simulation grids. 
Observation 19 The detailed experimental analysis indicated that the proposed model is found to be superior to ACO algorithm under all the applied scenarios. Table 7 illustrates some of the cases, the ACO algorithm has exhibited closer results to proposed model, but not better than the performance of the proposed models. The proposed method has attained better performance due to the merits of SSO, binary SSO and modified SSO algorithms. For proposed scheme such as sampling-based estimation scheme (SES), binary SSO, modified SSO. M-SSO for efficient cluster head selection in VANET is illustrated with result comparison. The optimal result is selected based on the complete coverage of all vehicles in VANET scenario as a secondary factor. The quality assessment has been made in terms of number of clusters under different test bed scenarios. There exists a consistent performance in the proposed algorithm on comparing the results with existing algorithms.

\section{Conclusion}

The issues and challenges, such as security-related issues, communication issues and topological issues, arise as the applicability of ad hoc network increases. In the proposed article, as a milestone approach to cluster the vehicles after choosing appropriate cluster head. By considering a graph ' $G$ ' which consists of a vehicle ' $V$ ' with its own communication range and covering range to overcome the data delivery rate and throughput. Moreover, the design imposed of an improved bio-inspired binary social spider algorithm, which works as a colony to achieve the global minimum points by concentrating on cooperative behavior of social spider algorithm. The proposed model outperforms the ACO algorithm with an increase in the transmission range. In case of low transmission range, the ACO and proposed algorithms have exhibited closer results and the proposed algorithm tends to show superior results to other methods under maximum transmission range. This research work has focused on designing and developing an effective bio-inspired social spider algorithm for solving clustering and data dissemination in VANET with the aim of improving on the weak performance of the existing models by designing an efficient binary social spider algorithm for effective clustering in VANET. The proposed methodology has been extended to support effective data dissemination with the clusters formed. The research results presented herein may be used to extend the field of research applications, to increase the clustering of more vehicles in a group and to improve the efficiency in traffic management, in order to offer reliable assistance for drivers and enhance the modern field of vehicle design.

\section{Declarations}

Conflict of interest There is no conflict of interest.

\section{References}

Leal, M. A., Matthias R., Bernhard K., Fabian de P. M., Thomas S (2010). Information-centric opportunistic data dissemination in vehicular ad hoc networks. In Intelligent Transportation Systems (ITSC), 2010 13th International IEEE Conference on, (pp. 1072-1078). IEEE.

Abkenar, F. S., \& GhaffarpourRahbar, A. (2017). Study and analysis of routing and spectrum allocation (rsa) and routing, modulation and spectrum allocation (rmsa) algorithms in elastic optical networks (eons). Optical Switching and Networking, 23, 5-39. 
Alodadi, K., Al-Bayatti, A. H., \& Alalwan, N. (2017). Cooperative volunteer protocol to detect non-line of sight nodes in vehicular ad hoc networks. Vehicular Communications, 9, 72-82.

Azimi, R., Sajedi, H., \& Ghayekhloo, Ms. (2017). A distributed data clustering algorithm in P2P networks. Applied Soft Computing, 51, 147-167.

Babukarthik, R. G., Adiga, V. A. K., Sambasivam, G., Chandramohan, D., \& Amudhavel, J. (2020). Prediction of COVID-19 using genetic deep learning convolutional neural network (GDCNN). IEEE Access, 8, 177647-177666. https://doi.org/10.1109/ACCESS.2020.3025164

Bao, Xu., Li, H., Zhao, G., Chang, Lv., Zhou, J., \& Li, Y. (2020). Efficient clustering V2V routing based on PSO in VANETs. Measurement. https://doi.org/10.1016/j.measurement.2019.107306 ISSN 0263-2241.

Benslimane, A., Tarik, T., \& Rajarajan, S. (2011). Dynamic clustering-based adaptive mobile gateway management in integrated VANET-3G heterogeneous wireless networks. IEEE Journal on Selected Areas in Communications, 29(3), 559-570.

Bhosale, P., Amarsinh V (2016). An agglomerative approach to elect the cluster head in VANET. In Signal Processing, Communication, Power and Embedded System (SCOPES), 2016 International Conference on, (pp. 1340-1344). IEEE.

Chandramohan, D., Dumka, A., Dhilipkumar, V., \& Loganathan, J. (2020). Data dissemination for greenVANETs communication: an opportunistic optimization approach. International Journal of Pervasive Computing and Communications. https://doi.org/10.1108/IJPCC-04-2020-0030

Chandramohan, D., Dumka, A., \& Jayakumar, L. (2020). 2M2C-R2ED: Multi-metric cooperative clustering based routing for energy efficient data dissemination in green-VANETs. Technol Econ Smart Grids Sustain Energy, 5, 15. https://doi.org/10.1007/s40866-020-00086-4

Chauhan, S., Banerjee, R., Chinmay, C., Mittal, M., Shiva, A., \& Ravi, V. (2021). A self-congruence and impulse buying effect on user's shopping behaviour over social networking sites: An empirical study. Int J of Pervasive Computing and Communications, 17(4), 404-425. https://doi.org/10.1108/ IJPCC-01-2021-0013

Dhurandher, S. K., Deepak, K. S., Isaac, W., \& Shruti, B. (2013). Routing Protocols in Infrastructure-Less Opportunistic Networks. In Alagan Anpalagan, Athanasios V. Vasilakos, Isaac Woungang, \& Sanjay Kumar Dhurandher (Eds.), Routing in Opportunistic Networks (pp. 353-382). New York: Springer.

Dubois, D. J., Bando, Y., Watanabe, K., \& Holtzman, H. (2013). Lightweight self-organizing reconfiguration of opportunistic infrastructure-mode wifi networks. In 2013 IEEE 7th International Conference on Self-Adaptive and Self-Organizing Systems (pp. 247-256). IEEE.

Eappen, G., \& Shankar, T. (2020). Hybrid PSO-GSA for energy efficient spectrum sensing in cognitive radio network. Physical Communication. https://doi.org/10.1016/j.phycom.2020.101091 ISSN 1874-4907.

Fan, P., Haran, J., Dillenburg, J., \& Nelson, P. (2005). Cluster-based framework in vehicular ad-hoc networks. In Violet R. Syrotiuk \& Edgar Chávez (Eds.), Ad-hoc mobile and wireless networks (pp. 32-42). Berlin: Springer.

Gerla, M., \& Tsai, J.-C. (1995). Multicluster, mobile, multimedia radio network. Wireless Networks, 1(3), 255-265.

Hacioglu, G., Kand, V. F. A., \& Sesli, E. (2016). Multi objective clustering for wireless sensor networks. Expert Systems with Applications, 59, 86-100.

Jalalvandi, S., Reza R (2016). A cluster-based routing algorithm for VANET. In Computer and Communications (ICCC), 2016 2nd IEEE International Conference on, (pp. 2068-2072). IEEE.

Jianwen, N., Liu C., Ma J (2009). Opportunistic Auto-Adaptive Dissemination in Vehicular Ad-hoc Networks. In Networks and Communications, 2009. NETCOM'09. First International Conference on, (pp. 40-45). IEEE.

Jitendra, P., Abhaya, K. S., Subhendu, K. P., \& Chinmay, C. (2021). Elementary framework for an iot based diverse ambient air quality monitoring system. Springer Multimedia Tools and Appl. https://doi.org/10. 1007/s11042-021-11285-1

Khan, M. F., Aadil, F., Maqsood, M., Bukhari, S. H. R., Hussain, M., \& Nam, Y. (2019). Moth flame clustering algorithm for internet of vehicle (MFCA-IoV). IEEE Access, 7, 11613-11629. https://doi.org/ 10.1109/ACCESS.2018.2886420

Khelifi, H., Luo, S., Nour, B., Moungla, H., Faheem, Y., Hussain, R., \& Ksentini, A. (2020). Named data networking in vehicular ad hoc networks: State-of-the-art and challenges. In IEEE Communications Surveys and Tutorials, 22(1), 320-351. https://doi.org/10.1109/COMST.2019.2894816

Lee, YangMin. (2017). Classification of node degree based on deep learning and routing method applied for virtual route assignment. Ad Hoc Networks, 58, 70-85.

Marfia, G., Pau, G., De Sena, E., Giordano, E., \& Gerla, M. (2007). Evaluating vehicle network strategies for downtown Portland: Opportunistic infrastructure and the importance of realistic mobility models. In Proceedings of the 1st international MobiSys workshop on Mobile opportunistic networking (pp. 47-51). ACM. 
Maslekar, N., Mounir B., Joseph M., Houda L (2011). A stable clustering algorithm for efficiency applications in VANETs. In Wireless Communications and Mobile Computing Conference (IWCMC), 2011 7th International, (pp. 1188-1193). IEEE.

Merhi, Z., Oussama T., Samih A-N, Amin H-A, and Magdy B (2015) Decentralized clustering in VANET using adaptive resonance theory. In Electronics, Circuits, and Systems (ICECS), 2015 IEEE International Conference on, (pp. 205-208). IEEE.

Nyati, S. S., \& Limkar, S. V. (2013). Cooperative and opportunistic transmission in wireless and mobile adhoc networks. Journal of Advances in Computer Network. https://doi.org/10.7763/JACN.2013.V1.43

Oliveira, R., Montez, C., Boukerche, A., \& Wangham, M. S. (2017). Reliable data dissemination protocol for VANET traffic safety applications. Ad Hoc Networks, 63, 30-44.

Oubbati, O. S., Lakas, A., Zhou, F., Güneş, M., Lagraa, N., \& Yagoubi, M. B. (2017). Intelligent UAVassisted routing protocol for urban VANETs. Computer Communications, 107, 93-111.

Qing, H., Jing, X., Pengfei, L., Sun, Q., \& Huang, J. (2020). A novel carrier frequency offset algorithm based on a double Barker code in VDE-TER. Communication. https://doi.org/10.1016/j.phycom.2020. 101059 ISSN 1874-4907.

Sangare, M., Gupta, S., Bouzefrane, S., Banerjee, S., \& Muhlethaler, P. (2020). Exploring the forecasting approach for road accidents: Analytical measures with hybrid machine learning. Expert Systems with Applications. https://doi.org/10.1016/j.eswa.2020.113855 ISSN 0957-4174.

Seyyedabbasi, A., \& Kiani, F. (2020). MAP-ACO: An efficient protocol for multi-agent pathfinding in realtime WSN and decentralized IoT systems. Microprocessors and Microsystems. https://doi.org/10. 1016/j.micpro.2020.103325 ISSN 0141-9331.

Sharma, V., Abhinav V., Manish G (2016). AES based security clustering routing for VANET. In Signal Processing, Communication, Power and Embedded System (SCOPES), 2016 International Conference on, (pp. 332-336). IEEE.

Shi, J., Wang, X., Huang, M., Li, K., \& DasShi, S. K. (2017). Social-based routing scheme for fixed-line VANET. Computer Networks, 113, 230-243.

Song, C., Jie, W., Liu, M., \& Zheng, H. (2017). Efficient routing through discretization of overlapped road segments in VANETs. Journal of Parallel and Distributed Computing, 102, 57-70.

Souza, E., Ioanis N., Pawel G (2010). A new aggregate local mobility (ALM) clustering algorithm for VANETs. In Communications (ICC), 2010 IEEE International Conference on, (pp. 1-5). IEEE.

Su, H., \& Zhang, Xi. (2007). Clustering-based multichannel MAC protocols for QoSprovisionings over vehicular ad hoc networks. IEEE Transactions on Vehicular Technology, 56(6), 3309-3323.

Sunil, K. M., Manju, K., Chinmay, C., Deepak, S., \& Durgesh, S. (2021). Performance evaluation of AODV and DSR protocols of flying ad-hoc network using highway mobility model. J of Circuits Systems and Computers, 31(1), 1-31. https://doi.org/10.1142/S0218126622500086

Taleb, T., Abderrahim, B., \& Khaled, B. L. (2010). Toward an effective risk-conscious and collaborative vehicular collision avoidance system. IEEE Transactions on Vehicular Technology, 59(3), 1474-1486.

Tamang, J., De Dieu, J., Nkapkop, M. F., Ijaz, P. K., Prasad, N. T., Saha, A., Kengne, J., \& Son, Y. (2021). Dynamical properties of ion-acoustic waves in space plasma and its application to image encryption. In IEEE Access, 9, 18762-18782. https://doi.org/10.1109/ACCESS.2021.3054250

Ucar, S., Sinem C. E., Oznur O (2013). VMaSC: Vehicular multi-hop algorithm for stable clustering in vehicular ad hoc networks. In Wireless Communications and Networking Conference (WCNC), 2013 IEEE, (pp. 2381-2386). IEEE.

Wang, Z., Lichuan, L., Meng, C. Z., \& Nirwan, A. (2008). A position-based clustering technique for ad hoc intervehicle communication. IEEE Transactions on Systems, Man, and Cybernetics, Part C (Applications and Reviews), 38(2), 201-208.

Wolny, G (2008) Modified DMAC clustering algorithm for VANETs. In Systems and Networks Communications, 2008. ICSNC'08. 3rd International Conference on, (pp. 268-273). IEEE.

Yu, J. Y., and Peter HJ Chong (2003). 3hbac (3-hop between adjacent clusterheads): a novel non-overlapping clustering algorithm for mobile ad hoc networks. In Communications, Computers and signal Processing, 2003. PACRIM. 2003 IEEE Pacific Rim Conference on, vol. 1, (pp. 318-321). IEEE.

Zhang, Z., Azzedine B., Richard P (2011). A novel multi-hop clustering scheme for vehicular ad-hoc networks. In Proceedings of the 9th ACM international symposium on Mobility management and wireless access, (pp. 19-26). ACM.

Zhang, X., Zhang, X., \& Cheng, Gu. (2017). A micro-artificial bee colony based multicast routing in vehicular ad hoc networks. Ad Hoc Networks, 58, 213-221.

Publisher's Note Springer Nature remains neutral with regard to jurisdictional claims in published maps and institutional affiliations. 


\title{
Authors and Affiliations
}

\section{Achyut Shankar ${ }^{1} \cdot$ Rajaguru Dayalan $^{2} \cdot$ Chinmay Chakraborty $^{3}$.} Chandramohan Dhasarathan ${ }^{4} \cdot$ Manish Kumar $^{5}$

\author{
Achyut Shankar \\ ashankar2711@gmail.com
}

Rajaguru Dayalan

raja.gur42@gmail.com

Chandramohan Dhasarathan

pdchandramohan@gmail.com

Manish Kumar

mmk9309@gmail.com

1 Department of Computer Science and Engineering, ASET, Amity University, Noida, Uttar Pradesh, India

2 Department of Computer Science \& Engineering, Pondicherry University, Puducherry, India

3 Department of CSE, Birla Institute of Technology, Jharkhand, India

4 Department of Computer Science \& Engineering, Madanapalle Institute of Technology \& Science, Madanapalle, India

5 Department of Computer Science and Engineering, VIT Deemed To University, Chennai, India 